\title{
Nuclear Effects in Neutrino Interactions and Their Impact on the Determination of Oscillation Parameters
}

\author{
Omar Benhar and Noemi Rocco \\ INFN and Department of Physics, "Sapienza" Universitá di Roma, Piazzale Aldo Moro 2, 00185 Roma, Italy \\ Correspondence should be addressed to Omar Benhar; omar.benhar@romal.infn.it
}

Received 30 July 2013; Accepted 12 October 2013

Academic Editor: Srubabati Goswami

Copyright (C) 2013 O. Benhar and N. Rocco. This is an open access article distributed under the Creative Commons Attribution License, which permits unrestricted use, distribution, and reproduction in any medium, provided the original work is properly cited.

\begin{abstract}
The quantitative description of the effects of nuclear dynamics on the measured neutrino-nucleus cross sections-needed to reduce the systematic uncertainty of long baseline neutrino oscillation experiments-involves severe difficulties. Owing to the uncertainty on the incoming neutrino energy, different reaction mechanisms contribute to the cross section measured at fixed energy and scattering angle of the outgoing lepton, and must therefore be consistently taken into account within a unified model. We research the theoretical approach based on the impulse approximation and the use of realistic nucleon spectral functions, allowing one to describe a variety of reaction mechanisms active in the broad kinematical range covered by neutrino experiments. The extension of this scheme to include more complex mechanisms involving the two-nucleon currents, which are believed to be important, is also outlined. The impact of nuclear effects on the determination of neutrino oscillation parameters is illustrated by analyzing the problem of neutrino energy reconstruction.
\end{abstract}

\section{Introduction}

Experimental searches of neutrino oscillations exploit neutrino-nucleus interactions to infer the properties of the beam particles, which are largely unknown. The use of nuclear targets as detectors, while allowing for a substantial increase of the event rate, entails nontrivial problems, as the interpretation of the observed signal requires a quantitative understanding of neutrino-nucleus interactions. Given the present experimental accuracy, the treatment of nuclear effects is in fact regarded as one of the main sources of systematic uncertainty (see, e.g., [1]).

Over the past decade, a growing effort has been made, aimed at making use of the knowledge of the nuclear response acquired from experimental and theoretical studies of electron scattering. Electron-nucleus scattering cross-sections are usually analyzed at fixed beam energy $E_{e}$, and electron scattering angle $\theta_{e}$ as a function of the energy loss $\omega$. As an example, Figure 1 shows the typical behavior of the double differential cross-section of the following inclusive process:

$$
e+A \longrightarrow e^{\prime}+X
$$

in which only the outgoing lepton is detected, at beam energy around $1 \mathrm{GeV}$. Here, $A$ and $X$ denote the target nucleus, in its ground state, and the undetected hadronic final state, respectively.

It is apparent that the different reaction mechanisms, yielding the dominant contributions to the cross-section at different values of $\omega$ (corresponding to different values of the Bjorken scaling variable $x=Q^{2} / 2 M \omega$, where $M$ is the nucleon mass and $Q^{2}=4 E_{e}\left(E_{e}-\omega\right) \sin ^{2} \theta_{e} / 2$ can be easily identified.

The bump centered at $\omega \sim Q^{2} / 2 M$, or $x \sim 1$, the position and width of which are determined by the momentum and removal energy distribution of the struck particle, corresponds to single nucleon knockout, while the structure visible at larger $\omega$ reflects the onset of coupling to two-nucleon currents, arising from meson exchange processes, excitation of nucleon resonances, and deep inelastic scattering.

The available theoretical models of electron-nucleus scattering provide an overall satisfactory description of the data over a broad kinematical range. In particular, in the region in which quasielastic scattering dominates, the data is generally reproduced with an accuracy of few percentages (for a recent 


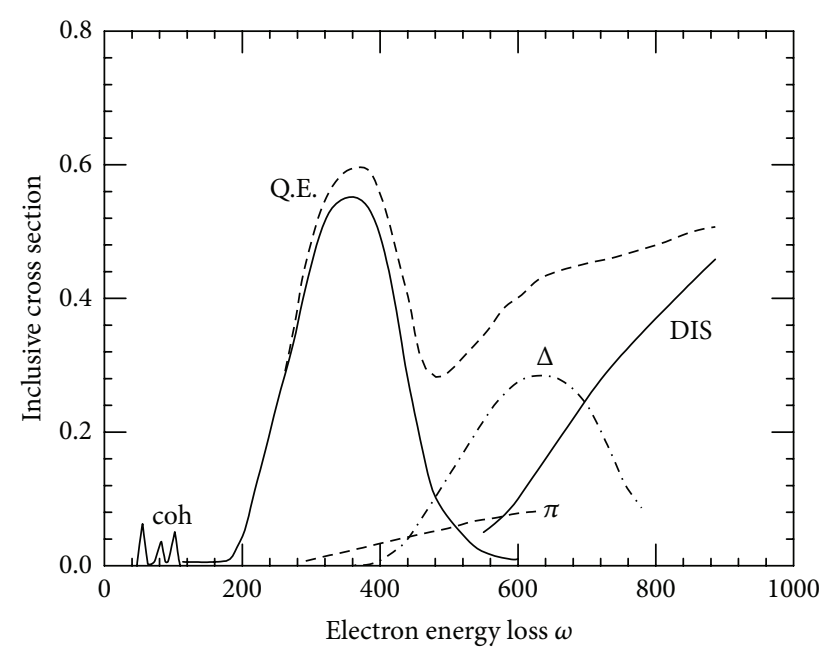

FIGURE 1: Schematic representation of the inclusive electron-nucleus cross-section at beam energy around $1 \mathrm{GeV}$, as a function of energy loss.

review on electron-nucleus scattering in the quasielastic sector, see [2]).

Because neutrino beams are always produced as secondary decay products, their energy is not sharply defined, but broadly distributed. As a consequence, in chargedcurrent neutrino scattering processes detecting the energy of the outgoing lepton, $T_{\ell}$, does not provide a measurement of the energy transfer, $\omega$, and different reaction mechanisms can contribute to the double differential cross-section at fixed $T_{\ell}$ and lepton scattering angle, $\theta_{\ell}$. This feature is illustrated in the Figure 2(a), showing the inclusive electron-carbon cross-sections at $\theta_{e}=37^{\circ}$ and beam energies ranging between 0.730 and $1.501 \mathrm{GeV}$, as a function of energy of the outgoing electron $[3,4]$. It clearly appears that the highlighted $550<T_{e^{\prime}}<650 \mathrm{MeV}$ bin, corresponding to quasifree kinematics at $E_{e}=730 \mathrm{MeV}$, picks up contributions from scattering processes taking place at different beam energies, in which reaction mechanisms other than single nucleon knockout are known to be dominant. To gauge the extent to which different contributions are mixed up in a typical neutrino experiment, consider the energy distribution of the MiniBooNE neutrino flux, displayed in Figure 2(b), showing that the fluxes corresponding to energies $E_{v}=730$ and $961 \mathrm{MeV}$ are within less than $20 \%$ of one another. It follows that, if we were to average the electron-carbon data of the left panel with the flux of the right panel, the cross-sections corresponding to beam energies 730 and $961 \mathrm{MeV}$ would contribute to the measured cross-section in the highlighted bin with about the same weight.

The above discussion implies that the understanding of the flux averaged neutrino cross-section requires the development of theoretical models providing a consistent treatment of all reaction mechanisms active in the broad kinematical range corresponding to the relevant neutrino energies.

In Section 2, we discuss the structure of the neutrinonucleus cross-section and point out that a consistent treatment of relativistic effects and nucleon-nucleon correlations requires the factorization of the nuclear vertex. The main elements of the resulting expression of the cross-section, that is, the nucleon spectral function and the elementary neutrino-nucleon cross-section, are also analyzed. In Section 3, we briefly research the available empirical information on the nucleon weak structure functions in the kinematical regimes corresponding to quasielastic scattering, resonance production, and deep inelastic scattering, while Section 4 is devoted to a discussion of the ambiguities implied in the interpretation of the events labeled as quasielastic. As an example of the impact of nuclear effects on the determination of neutrino oscillations, in Section 5 we analyze the problem of neutrino energy reconstruction. Finally, in Section 6 we summarize the main issues and state our conclusions.

\section{The Neutrino-Nucleus Cross-section}

Let us consider, for definiteness, charged-current neutrinonucleus interactions. The formalism discussed in this section can be readily generalized to the case of neutral current interactions [6]. The double differential cross-section of the process (compare to (1))

$$
v_{\ell}+A \longrightarrow \ell^{-}+X
$$

can be written in the following form [7]:

$$
\frac{d^{2} \sigma}{d \Omega_{\mathbf{k}^{\prime}} d k_{0}^{\prime}}=\frac{G_{F}^{2} V_{u d}^{2}}{16 \pi^{2}} \frac{\left|\mathbf{k}^{\prime}\right|}{|\mathbf{k}|} L_{\mu \nu} W_{A}^{\mu \nu} .
$$

In the above equation, $k \equiv\left(k_{0}, \mathbf{k}\right)$ and $k^{\prime} \equiv\left(k_{0}^{\prime}, \mathbf{k}^{\prime}\right)$ are the four momenta carried by the incoming neutrino and the outgoing charged lepton, respectively, $G_{F}$ is the Fermi constant, and $V_{u d}$ is the CKM matrix element coupling $u$ and $d$ quarks. The tensor $L_{\mu \nu}$, defined as (we neglect the term proportional to $m_{\ell}^{2}$, where $m_{\ell}$ is the mass of the charged lepton)

$$
L_{\mu \nu}=8\left[k_{\mu}^{\prime} k_{\nu}+k_{\nu}^{\prime} k_{\mu}-g_{\mu \nu}\left(k \cdot k^{\prime}\right)-i \varepsilon_{\mu \nu \alpha \beta} k^{\prime \beta} k^{\alpha}\right],
$$

is completely determined by the lepton kinematics, whereas the nuclear tensor $W_{A}^{\mu \nu}$, containing all the information on strong interaction dynamics, describes the response of the target nucleus. Its definition

$$
W_{A}^{\mu \nu}=\sum_{x}\left\langle 0\left|J_{A}^{\mu \dagger}\right| X\right\rangle\left\langle X\left|J_{A}^{\nu}\right| 0\right\rangle \delta^{(4)}\left(p_{0}+q-p_{X}\right),
$$

with $q=k-k^{\prime}$, involves the target initial and final states $|0\rangle$ and $|X\rangle$, carrying four momenta $p_{0}$ and $p_{X}$, respectively, as well as the following nuclear current operator:

$$
J_{A}^{\mu}=\sum_{i} j_{i}^{\mu}+\sum_{j>i} j_{i j}^{\mu}
$$

where $j_{i j}^{\mu}$ denotes the two-nucleon contribution arising from meson-exchange processes.

In the kinematical region corresponding to low momentum transfer, typically $|\mathbf{q}|<400 \mathrm{MeV}$, in which nonrelativistic approximations are expected to work, the tensor of (5) can be 


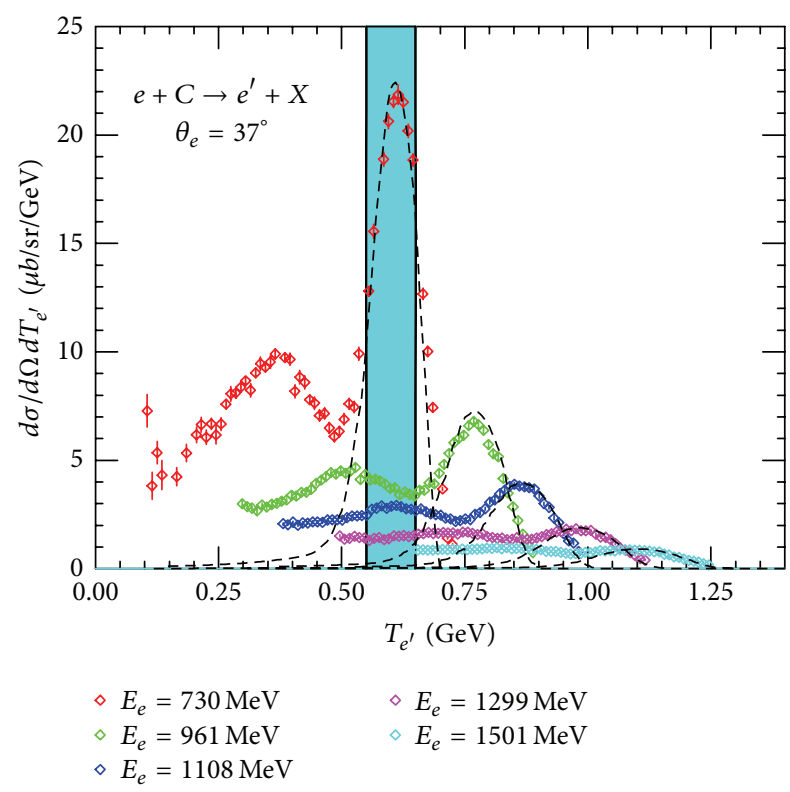

(a)

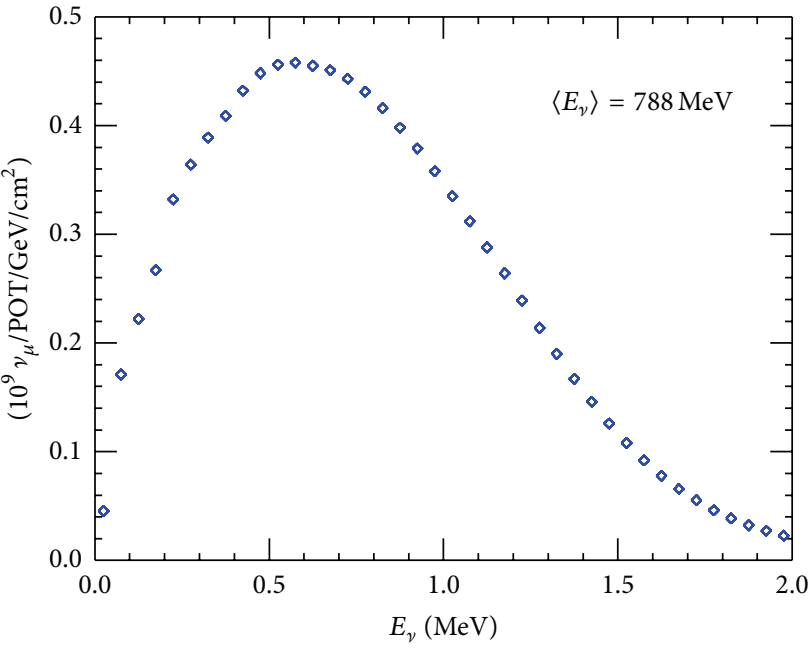

(b)

Figure 2: (a) Inclusive electron-carbon cross-sections at $\theta_{e}=37^{\circ}$ and beam energies ranging between 0.730 and $1.501 \mathrm{GeV}$ [3, 4], plotted as a function of the energy of the outgoing electron. (b) Energy dependence of the MiniBooNE neutrino flux [5].

evaluated within highly realistic nuclear models $[8,9]$. However, the event analysis of accelerator-based neutrino experiments requires theoretical approaches that can be applied in the relativistic regime. The importance of relativistic effects can be easily grasped considering that the mean momentum transfer of quasielastic (QE) processes obtained by averaging over the MiniBooNE [5] and Minerva [10] neutrino fluxes turn out to be $\sim 640$ and $\sim 880 \mathrm{MeV}$, respectively.

Nonrelativistic nuclear many-body theory, based on dynamical models strongly constrained by phenomenology, provides a fully consistent theoretical approach allowing for an accurate description of the target initial state, independent of momentum transfer. On the other hand, at large $|\mathbf{q}|$, the treatment of both the nuclear current and the hadronic final state unavoidably requires approximations.

2.1. The Impulse Approximation. The Impulse Approximation (IA) scheme, extensively employed to analyze electronnucleus scattering data [2], is based on the tenet that, at momentum transfer $\mathbf{q}$ such that $\mathbf{q}^{-1} \ll d$, $d$ being the average nucleon-nucleon distance in the target, neutrinonucleus scattering reduces to the incoherent sum of scattering processes involving individual nucleons. Moreover, final state interactions between the outgoing hadrons and the spectator nucleons are assumed to be negligible.

Within the IA picture, the nuclear current of (6) reduces to the sum of one-body terms, while the final state simplifies to the direct product of the hadronic state produced at the interaction vertex, with momentum $\mathbf{p}_{x}$, and the state describing the $(A-1)$-nucleon residual system, carrying momentum $\mathbf{p}_{R}$, that is,

$$
|X\rangle \longrightarrow\left|x, \mathbf{p}_{x}\right\rangle \otimes\left|R, \mathbf{p}_{R}\right\rangle,
$$

implying

$$
\begin{aligned}
\sum_{X}|X\rangle\langle X| \longrightarrow & \sum_{x} \int d^{3} p_{x}\left|x, \mathbf{p}_{x}\right\rangle\left\langle\mathbf{p}_{x}, x\right| \\
& \times \sum_{R} \int d^{3} p_{R}\left|R, \mathbf{p}_{R}\right\rangle\left\langle\mathbf{p}_{R}, R\right|
\end{aligned}
$$

Insertion of a complete set of free nucleon states, satisfying

$$
\int d^{3} k|N, \mathbf{k}\rangle\langle\mathbf{k}, N|=1,
$$

leads to the factorization of the nuclear current matrix element according to

$$
\begin{aligned}
\left\langle 0\left|J_{A}^{\mu}\right| X\right\rangle= & \left(\frac{M}{\sqrt{\left|\mathbf{p}_{R}\right|^{2}+M^{2}}}\right)^{1 / 2}\left\langle 0 \mid R, \mathbf{p}_{R} ; N,-\mathbf{p}_{R}\right\rangle \\
& \times \sum_{i}\left\langle-\mathbf{p}_{R}, N\left|j_{i}^{\mu}\right| x, \mathbf{p}_{x}\right\rangle,
\end{aligned}
$$

where the factor $\left(M / \sqrt{\left|\mathbf{p}_{R}\right|^{2}+M^{2}}\right)^{1 / 2}$, with $M$ being the nucleon mass, takes into account the implicit covariant normalization of the state $\left\langle-\mathbf{p}_{R}, N\right|$ in the matrix element of $j_{i}^{\mu}$. 
Using the above relations, the hadronic tensor can be rewritten in the following form:

$$
\begin{aligned}
W^{\mu \nu}=\sum_{R, x} \int & d^{3} p_{R} d^{3} p_{x}\left|\left\langle 0 \mid R, \mathbf{p}_{R} ; N,-\mathbf{p}_{R}\right\rangle\right|^{2} \\
& \times\left(\frac{M}{\sqrt{\left|\mathbf{p}_{R}\right|^{2}+M^{2}}}\right) \\
& \times \sum_{i}\left\langle-\mathbf{p}_{R}, N\left|j_{i}^{\mu \dagger}\right| x, \mathbf{p}_{x}\right\rangle\left\langle\mathbf{p}_{x}, x\left|j_{i}^{\nu}\right| N,-\mathbf{p}_{R}\right\rangle \\
& \times \delta^{3}\left(\mathbf{q}-\mathbf{p}_{R}-\mathbf{p}_{x}\right) \delta\left(\omega+E_{0}-E_{R}-E_{x}\right),
\end{aligned}
$$

where $E_{0}$ is the target ground state energy, $E_{R}=$ $\sqrt{\left|\mathbf{p}_{R}\right|^{2}+M_{R}^{2}}, M_{R}$ being the mass of the recoiling system, and $E_{x}$ is the energy of the hadronic state $x$ produced at the interaction vertex.

Equation (11) can be cast in the following more concise form:

$$
\begin{aligned}
W_{A}^{\mu \nu}= & \int d^{3} p d E P(E, \mathbf{p}) \frac{M}{E_{p}} \\
& \times \sum_{x}\left\langle\mathbf{p}, N\left|j_{i}^{\mu \dagger}\right| x, \mathbf{p}+\mathbf{q}\right\rangle\left\langle x, \mathbf{p}+\mathbf{q}\left|j_{i}^{v}\right| \mathbf{p}, N\right\rangle \\
& \times \delta\left(\widetilde{\omega}+\sqrt{\mathbf{p}^{2}+M^{2}}-E_{x}\right),
\end{aligned}
$$

where

$$
\begin{aligned}
\widetilde{\omega} & =E_{x}-\sqrt{\mathbf{p}^{2}+M^{2}}=\omega+M-E-\sqrt{\mathbf{p}^{2}+M^{2}} \\
& =\omega+E_{0}-E_{R}-\sqrt{\mathbf{p}^{2}+M^{2}},
\end{aligned}
$$

and the spectral function

$$
P(\mathbf{p}, E)=\sum_{R}|\langle 0 \mid R,-\mathbf{p}\rangle|^{2} \delta\left(E-M+E_{0}-E_{R}\right)
$$

yields the probability of removing a nucleon with momentum p from the target ground state leaving the residual system with excitation energy $E$.

Using the definition of the tensor describing the interactions of the $i$ th nucleon in free space (the subscripts $\alpha=p, n$ denote proton and neutron, resp.)

$$
\begin{gathered}
\mathscr{W}_{\alpha}^{\mu \nu}=\sum_{x}\left\langle\mathbf{p}, N\left|j_{\alpha}^{\mu \dagger}\right| x, \mathbf{p}+\mathbf{q}\right\rangle\left\langle x, \mathbf{p}+\mathbf{q}\left|j_{\alpha}^{\nu}\right| \mathbf{p}, N\right\rangle \\
\times \delta\left(\widetilde{\omega}+\sqrt{\mathbf{p}^{2}+M^{2}}-E_{x}\right),
\end{gathered}
$$

we finally obtain, for a target nucleus with $Z=A / 2$,

$$
W_{A}^{\mu \nu}=A \int d^{3} p d E \frac{M}{E_{p}} P(\mathbf{p}, E) \mathscr{W}_{N}^{\mu \nu},
$$

with $\mathscr{W}_{N}^{\mu \nu}=\left(\mathscr{W}_{p}^{\mu \nu}+\mathscr{W}_{n}^{\mu \nu}\right) / 2$. Note that in deriving the above equation, we have made the assumption, largely justified in isoscalar nuclei, that the proton and neutron spectral functions be the same.

It has to be emphasized that the replacement of $\omega$ with $\widetilde{\omega}$ (see (15)) is meant to take into account the fact that a fraction $\delta \omega$ of the energy transfer goes into excitation energy of the spectator system. Therefore, the elementary scattering process can be described as if it took place in free space with energy transfer $\widetilde{\omega}=\omega-\delta \omega$.

Collecting the above results, the nuclear cross-section can be finally written in the following transparent form:

$$
\frac{d^{2} \sigma_{\mathrm{IA}}}{d \Omega_{\mathbf{k}^{\prime}} d k_{0}^{\prime}}=A \int d^{3} p d E P(p, E) \frac{d^{2} \sigma_{\text {elem }}}{d \Omega_{\mathbf{k}^{\prime}} d k_{0}^{\prime}},
$$

with

$$
\frac{d^{2} \sigma_{\text {elem }}}{d \Omega_{\mathbf{k}^{\prime}} d k_{0}^{\prime}}=\frac{G_{F}^{2} V_{u d}^{2}}{16 \pi^{2}} \frac{\left|\mathbf{k}^{\prime}\right|}{|\mathbf{k}|} \frac{1}{4 E_{|\mathbf{p}|} E_{|\mathbf{p}+\mathbf{q}|}} L_{\mu \nu} \mathscr{W}_{N}^{\mu \nu} .
$$

In conclusion, within the IA scheme it is possible to trace back the hadronic tensor corresponding to the nuclear target to the ones describing the elementary interaction with isolated nucleons-which can be, at least in principle, measured using proton and deuteron targets-provided that the four momentum transfer $q$ is replaced with $\widetilde{q} \equiv(\widetilde{\omega}, \mathbf{q})$ and an integration on the nucleon momentum and removal energy is carried out, with a weight given by the spectral function.

We emphasize that, as will be discussed below, (17) and (18) can be applied to a variety of reaction mechanisms, including QE scattering, resonance production, and Deep Inelastic Scattering (DIS).

2.2. Spectral Function. The calculation of the target spectral function requires a model of nuclear dynamics. The simulation codes employed for the analysis of neutrino oscillation experiments are largely based on the relativistic Fermi gas model (RFGM) [11], according to which the target nucleus can be described as a degenerate gas of protons and neutrons obeying on-shell relativistic kinematics. Nucleons occupy all states with momenta smaller than the Fermi momentum $p_{F}$-belonging to the Fermi sea-and are bound with constant energy $\epsilon$. The values of these two parameters are determined through a fit of the position and width of the quasielastic peak of the measured electron-nucleus scattering cross-sections $[12,13]$.

Within the RFGM, the nuclear spectral function, defined in (14), can be written in the following simple form:

$$
P_{\mathrm{RFGM}}(p, E)=\frac{6 \pi^{2}}{p_{F}^{3}} \theta\left(p_{F}-p\right) \delta\left(E_{p}-\epsilon+E\right),
$$

where $E_{p}=\sqrt{|\mathbf{p}|^{2}+M^{2}}$.

Electron scattering data have provided overwhelming evidence that the energy-momentum distribution of nucleons in the nucleus is quite different from the one predicted by the RFGM. The differences are to be ascribed to the presence of nucleon-nucleon (NN) correlations, mainly arising from the strongly repulsive nature of the $\mathrm{NN}$ interactions at short distances. Dynamical correlations give rise to virtual scattering processes leading to the excitation of the participating 
nucleons to states of energy larger than the Fermi energy, thus depleting the single particle levels within the Fermi sea. Owing to the contribution of nucleons belonging to a correlated pair, the nuclear spectral function $P(\mathbf{p}, E)$ exhibits tails extending to the regions $|p| \gg p_{F}$ and $E \gg \epsilon$.

Highly accurate theoretical calculations of the spectral function can be carried out for uniform nuclear matter, exploiting the simplifications arising from translation invariance [14]. The results of these calculations have been combined with the information obtained from coincidence $\left(e, e^{\prime} p\right)$ experiments at moderate energy and momentum transfer, to obtain spectral functions of a variety of nuclei within the local density approximation (LDA) $[15,16]$.

According to the LDA scheme, the spectral function is written in the following form:

$$
P_{\mathrm{LDA}}(\mathbf{p}, E)=P_{\mathrm{MF}}(\mathbf{p}, E)+P_{\text {corr }}(\mathbf{p}, E),
$$

where the two terms describe the contributions associated with the nuclear mean field and NN correlations, respectively. The former is usually written in the following factorized form:

$$
P_{\mathrm{MF}}(\mathbf{p}, E)=\sum_{n \in\{F\}} Z_{n}\left|\phi_{n}(\mathbf{p})\right|^{2} F_{n}\left(E-E_{n}\right),
$$

where the sum is extended to all states belonging to the Fermi sea, while the spectroscopic factor $Z_{n}<1$ and the function $F_{n}\left(E-E_{n}\right)$, accounting for the finite width of the $n$th shell-model state, take into account the effects of residual interactions that are not included in the mean-field picture. In the absence of all interactions, $Z_{n} \rightarrow 1$ and $F\left(E-E_{n}\right) \rightarrow$ $\delta\left(E-E_{n}\right)$.

The correlation contribution is given by

$$
P_{\text {corr }}(\mathbf{p}, E)=\int d^{3} r \varrho_{A}(\mathbf{r}) P_{\text {corr }}^{\mathrm{NM}}\left(\mathbf{p}, E ; \varrho=\varrho_{A}(\mathbf{r})\right),
$$

where $\varrho_{A}(\mathbf{r})$ is the nuclear density distribution and $P_{\text {corr }}^{\mathrm{NM}}(\mathbf{p}$, $E ; \varrho)$ is the correlation part of the spectral function of nuclear matter at uniform density $\varrho$. Note that the spectroscopic factors $Z_{n}$ are constrained by the following normalization requirement:

$$
\int d^{3} p d E P_{\mathrm{LDA}}(\mathbf{p}, E)=1 .
$$

Typically, the mean-field contribution accounts for $\sim 80 \%$ of the above normalization integral. The correlation strength located at large $p$ and $E$ has been recently measured at JLab using a Carbon target [17]. The results of this analysis are consistent with the data at low missing energy and missing momentum, as well as with the results of theoretical calculations carried out within nuclear many-body theory.

2.3. Neutrino-Nucleon Vertex. The most general expression of the target tensor of (15) can be written in terms of five structure functions, depending on the Lorentz scalars $q^{2}$ and $(p \cdot q)$ only, as

$$
\begin{aligned}
\mathscr{W}^{\mu \nu}= & -g^{\mu \nu} W_{1}\left(q^{2},(p \cdot q)\right)+\frac{p^{\mu} p^{\nu}}{M^{2}} W_{2}\left(q^{2},(p \cdot q)\right) \\
& -i \epsilon^{\mu \nu \rho \sigma} \frac{p_{\varrho} q_{\sigma}}{2 M^{2}} W_{3}\left(q^{2},(p \cdot q)\right)+\frac{q^{\mu} q^{\nu}}{M^{2}} W_{4}\left(q^{2},(p \cdot q)\right) \\
& +\frac{p^{\mu} q^{\nu}+p^{\nu} q^{\mu}}{M^{2}} W_{5}\left(q^{2},(p \cdot q)\right) .
\end{aligned}
$$

In scattering processes involving isolated nucleons, the structure functions $W_{4}$ and $W_{5}$ give vanishing contributions to the cross-section, after contraction of the target tensor with $L_{\mu \nu}$. Owing to the replacement $q \rightarrow \widetilde{q}$ in the arguments of $\mathscr{W}^{\mu \nu}$, dictated by the IA, in neutrino-nucleus scattering, this is no longer the case. However, the results of numerical calculations suggest that the contribution of the terms involving $W_{4}$ and $W_{5}$ is small, and can be safely neglected [7].

The contraction of the above tensor with $L_{\mu \nu}$ of (4) can be cast in the following form:

$$
L^{\mu \nu} W_{\mu \nu}=16 \sum_{i} W_{i}\left(\frac{A_{i}}{M^{2}}\right),
$$

with the kinematical factors $A_{i}$ given by the following expressions:

$$
\begin{aligned}
& A_{1}=M^{2}\left(k \cdot k^{\prime}\right), \\
& A_{2}=(k \cdot p)\left(k^{\prime} \cdot p\right)-\frac{A_{1}}{2}, \\
& A_{3}=(k \cdot p)\left(k^{\prime} \cdot \tilde{q}\right)-(k \cdot \widetilde{q})\left(k^{\prime} \cdot p\right), \\
& A_{4}=(k \cdot \tilde{q})\left(k^{\prime} \cdot \tilde{q}\right)-\frac{\widetilde{q}^{2}}{2} \frac{A_{1}}{M^{2}}, \\
& A_{5}=(k \cdot p)\left(k^{\prime} \cdot \tilde{q}\right)+\left(k^{\prime} \cdot p\right)(k \cdot \tilde{q})-(\tilde{q} \cdot p) \frac{A_{1}}{M^{2}} .
\end{aligned}
$$

\section{Nucleon Structure Functions}

As already stated, the formalism based on the IA provides a unified framework, suitable to describe neutrino-nucleus interaction in different kinematical regimes. In this Section, we discuss the form of the structure functions $W_{i}$ in the $\mathrm{QE}$ and DIS regimes, and briefly outline the extension of the $\mathrm{QE}$ form to the case of resonance production.

3.1. Charged-Current Quasielastic (CCQE) Scattering and Resonance Production. In the CCQE channel, the structure functions involve the energy conserving $\delta$-function enforcing the condition that the scattering process be elastic. They are conveniently written in the following form:

$$
W_{i}=\widetilde{W}_{i} \delta\left(\widetilde{\omega}+\frac{\widetilde{q}^{2}}{2 M}\right),
$$


where the $\widetilde{W}_{i}$ can be obtained from the matrix elements of the nucleon current. Exploiting the CVC hypothesis and PCAC, the resulting structure functions, can be written in terms of the electromagnetic form factors $F_{1}$ and $F_{2}$ and the axial form factor $F_{A}$ according to

$$
\begin{aligned}
& \widetilde{W}_{1}=2\left[F_{A}^{2}(1+\tau)+\tau\left(F_{1}+F_{2}\right)^{2}\right], \\
& \widetilde{W}_{2}=2\left[F_{A}^{2}+F_{1}^{2}+\tau F_{2}^{2}\right], \\
& \widetilde{W}_{3}=2 F_{A}\left(F_{1}+F_{2}\right), \\
& \widetilde{W}_{4}=\frac{\left[F_{2}^{2}(1+\tau)-2 F_{2}\left(F_{1}+F_{2}\right)\right]}{2}, \\
& \widetilde{W}_{5}=\frac{W_{2}}{2},
\end{aligned}
$$

with $\tau=-q^{2} / 4 M^{2}$.

The form factors appearing in the vector current, $F_{1}\left(q^{2}\right)$ and $F_{2}\left(q^{2}\right)$, are obtained from the measured electric and magnetic nucleon form factors, $G_{E}$ and $G_{M}$, through the following relations:

$$
\begin{aligned}
& F_{1}\left(q^{2}\right)=\frac{1}{(1-\tau)}\left[G_{E}\left(q^{2}\right)-\tau G_{M}\left(q^{2}\right)\right], \\
& F_{2}\left(q^{2}\right)=\frac{1}{(1-\tau)}\left[-G_{E}\left(q^{2}\right)+G_{M}\left(q^{2}\right)\right] .
\end{aligned}
$$

While more refined parameterizations of the large body of data are available (for a review, see, e.g., [18]), the form factors $G_{E}$ and $G_{M}$ are often written in the following simple dipole form:

$$
\begin{gathered}
G_{E}\left(q^{2}\right)=\left(1-\frac{q^{2}}{M_{V}^{2}}\right)^{-2}, \\
G_{M}\left(q^{2}\right)=\left(\mu_{p}-\mu_{n}\right)\left(1-\frac{q^{2}}{M_{V}^{2}}\right)^{-2},
\end{gathered}
$$

with $M_{V}^{2}=0.71 \mathrm{GeV}^{2}$. The axial form factor, $F_{A}$, is also written in the same form as follows:

$$
F_{A}\left(q^{2}\right)=g_{A}\left(1-\frac{q^{2}}{M_{A}^{2}}\right)^{-2}
$$

The value of the axial coupling constant, $g_{A}=-1.261 \pm$ 0.004 , is obtained from neutron $\beta$-decay, while the axial mass extracted from low-statistics neutrino-deuteron scattering data is $M_{A}=1.032 \pm 0.036 \mathrm{GeV}[19,20]$. The contribution of the pseudoscalar form factor, $F_{P}$, can be safely neglected, except for the case of $\nu_{\tau}$ scattering.

The generalization of the above formalism to describe the resonance production region only involves minor changes. Unlike the CCQE case, the structure functions depend on both $q^{2}$ and $W^{2}$, the squared invariant mass of the hadronic final state, and the energy conserving $\delta$-function in (27) is replaced by the Breit-Wigner factor

$$
\frac{M_{R} \Gamma_{R}}{\pi} \frac{1}{\left(W^{2}-M_{R}^{2}\right)^{2}+M_{R}^{2} \Gamma_{R}^{2}},
$$

where $M_{R}$ and $\Gamma_{R}$ denote the resonance mass and its decay width, respectively. In addition, the nucleon form factors are replaced by the transition matrix elements of the nucleon weak current $[7,21]$.

The CCQE and resonance contributions to the total neutrino-nucleon cross-section reported by the authors of [7] are shown in Figure 3 as a function of neutrino energy. The resonance-production cross-section has been obtained taking into account both the $\Delta$ resonance and the three isospin $1 / 2$ states lying in the so-called second resonance region the $P_{11}(1440), D_{13}(1520)$, and $S_{11}(1535)$. It clearly appears that at beam energies $\sim 1 \mathrm{GeV}, \mathrm{QE}$ scattering and resonance production turn out to be comparable.

The decay of the $\Delta$ resonance is a prominent mechanism leading to the appearance of pions in the final state. A detailed discussion of both coherent and incoherent pion productions can be found in $[22,23]$.

3.2. Deep Inelastic Scattering. From the observational point of view, the DIS regime corresponds to hadronic final states with more than one pion.

In principle, the three nucleon structure functions entering the definition of the IA nuclear cross-section, (17) and (18), can be obtained combining neutrino and antineutrino scattering cross-sections. However, as the available structure functions have been extracted from nuclear cross-sections (see, e.g., [24]), their use in "bottom up" theoretical studies aimed at identifying nuclear effects involves obvious conceptual difficulties.

An alternative approach, allowing one to obtain the structure functions describing DIS on isolated nucleons, can be developed within the framework of the quark-parton model, exploiting the large database of DIS data collected using charged lepton beams and hydrogen and deuteron targets (see, e.g., [25]). Within this scheme, the function $F_{2}^{\nu N}=\omega W_{2}$, where $\omega$ is the energy transfer and $W_{2}$ is the weak structure function of an isoscalar nucleon, defined by (24), can be simply related to the corresponding structure function extracted from electron scattering data, $F_{2}^{e N}$, through

$$
F_{2}^{v N}=\frac{18}{5} F_{2}^{e N}
$$

In addition, the following relation:

$$
x \omega W_{3}=V(x),
$$

where $x$ is the Bjorken scaling variable and $V(x)$ denotes the valence quark distribution, implies that

$$
x \omega W_{3}=F_{2}^{e N}-2 \bar{q}(x),
$$

$\bar{q}(x)$ being the antiquark distribution.

Using the above results and the relation $F_{2}=2 x F_{1}$, with $F_{1}=M W_{1}$, one can readily obtain the weak structure functions from the existing parameterization of the electromagnetic structure functions and the antiquark distribution (see, e.g., [26]).

The above procedure rests on the tenet, underlying the IA scheme, that the elementary neutrino-nucleon interaction 


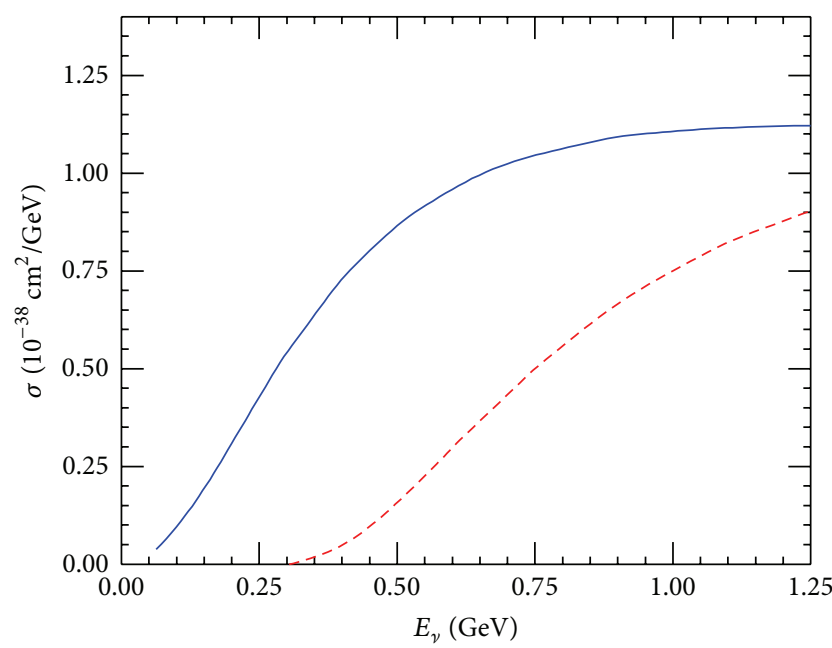

FIGURE 3: QE (solid line) and resonance production (dashed line) contributions to the charged-current neutrino-nucleon scattering cross-section (adapted from [7]).

is not affected by the presence of the nuclear medium. While this assumption is strongly supported by electronnucleus scattering data in the quasielastic channel, analyses of neutrino DIS data are often carried out allowing for a medium modification of the nucleon structure functions [27, 28], or of the parton distributions entering their definitions [29].

The approach of $[27,28]$ makes use of a model of the nuclear spectral function and includes a variety of medium effects, such as the $\pi$ - and $\rho$-meson cloud contributions and nuclear shadowing. The authors of [29], on the other hand, provide a parameterization of the nuclear parton distributions at order $\alpha_{s}$ obtained from a fit to the measured nuclear cross-sections.

\section{Interpretation of the CCQE Cross-Section}

The data set of CCQE events collected by the MiniBooNE collaboration [5] provides an unprecedented opportunity to carry out a systematic study of the double differential crosssection of the following process:

$$
\nu_{\mu}+{ }^{12} C \longrightarrow \mu^{-}+X,
$$

averaged over the neutrino flux shown in the right panel of Figure 2.

As pointed out in the previous Section, the CCQE neutrino-nucleon process is described in terms of three form factors. The proton and neutron electromagnetic form factors, which have been precisely measured up to large values of $Q^{2}$ in electron-proton and electron-deuteron scattering experiments, and the nucleon axial form factor $F_{A}$, parameterized in terms of the axial mass $M_{A}$ as in (31). The data analysis performed using the RFGM yields an axial mass $M_{A} \approx 1.35 \mathrm{GeV}$, significantly larger than that obtained from deuteron data $[19,20]$. A large value of the axial mass, $M_{A} \approx 1.2 \mathrm{GeV}$, has been also reported by the analysis of the CCQE neutrino-oxygen cross-section carried out by the $\mathrm{K} 2 \mathrm{~K}$ collaboration [30], while the NOMAD collaboration released the value $M_{A}=1.05 \mathrm{GeV}$, which is compatible with the world average of deuteron data, resulting from the analysis of CCQE neutrino- and antineutrino-carbon interactions at larger beam energies $\left(E_{v} \sim 10 \mathrm{GeV}\right)[31]$.

It would be tempting to interpret the value of $M_{A}$ reported by MiniBooNE as an effective axial mass, modified by nuclear effects not included in the RFGM. However, theoretical studies carried out within the IA scheme with a realistic carbon spectral function-an approach that has proved to be capable of providing a quantitative account of a wealth of electron scattering data in the quasielastic sector-fail to describe the flux averaged double differential cross-section of [5]. This striking feature is illustrated in Figure 4. The left panel shows a comparison between the electron scattering data of [3] and the results obtained using the spectral function of [15], while in the right panel the results obtained within the same scheme and setting $M_{A}=1.03 \mathrm{GeV}$ are compared to the flux averaged double differential CCQE cross-section measured by the MiniBooNE collaboration, shown as a function of kinetic energy of the outgoing muon [32]. It is apparent that height, position, and width of the QE peak measured in electron scattering, mostly driven by the energy and momentum dependence of the spectral function, are well reproduced, while the peaks exhibited by the neutrino crosssections are largely underestimated.

The authors of [32] argued that the differences observed in Figure 4 are to be largely ascribed to the flux average involved in the determination of the neutrino cross-section, leading to the appearance of contributions of reaction mechanisms not taken into account in the IA picture. To overcome this difficulty, they advocated the development of models based on a new paradigm, in which all relevant reaction mechanisms are consistently taken into account within a unified description of nuclear dynamics. While in this research, we will mainly focus on the approach based on the spectral function formalism, it has to be mentioned that a unified description of a variety of nuclear effects can be also obtained within a completely different framework, based on transport theory (for a recent review, see, e.g., [33]).

4.1. Role of Reaction Mechanisms other than Single Nucleon Knockout. In MiniBooNE data analysis, an event is labeled as CCQE if no final state pions are detected in addition to the outgoing muon. The simplest reaction mechanism compatible with this definition is single nucleon knockout, induced by the one-nucleon contributions to the nuclear current (see (6)). In the absence of $\mathrm{NN}$ correlations, the spectator $(A-1)$-particle system is left in a bound state, and the final nuclear state, consisting of the knocked out nucleon and the recoiling residual nucleus, is said to be a one particleone hole state.

It has been suggested that the observed excess of CCQE cross-section may be traced back to the occurrence of events with two particle-two hole final states [34-36]. According to the above definition, these events cannot be distinguished from those with one particle-one hole final states. Therefore, they are often referred to as CCQE-like. The role of two particle-two hole interactions at higher energies, up to $10 \mathrm{GeV}$, has also been recently discussed in [37]. 


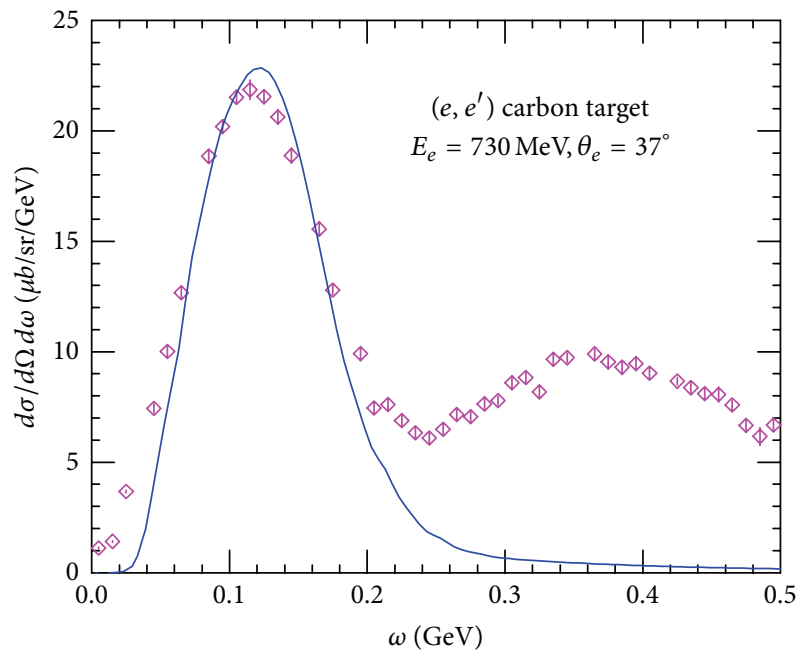

(a)

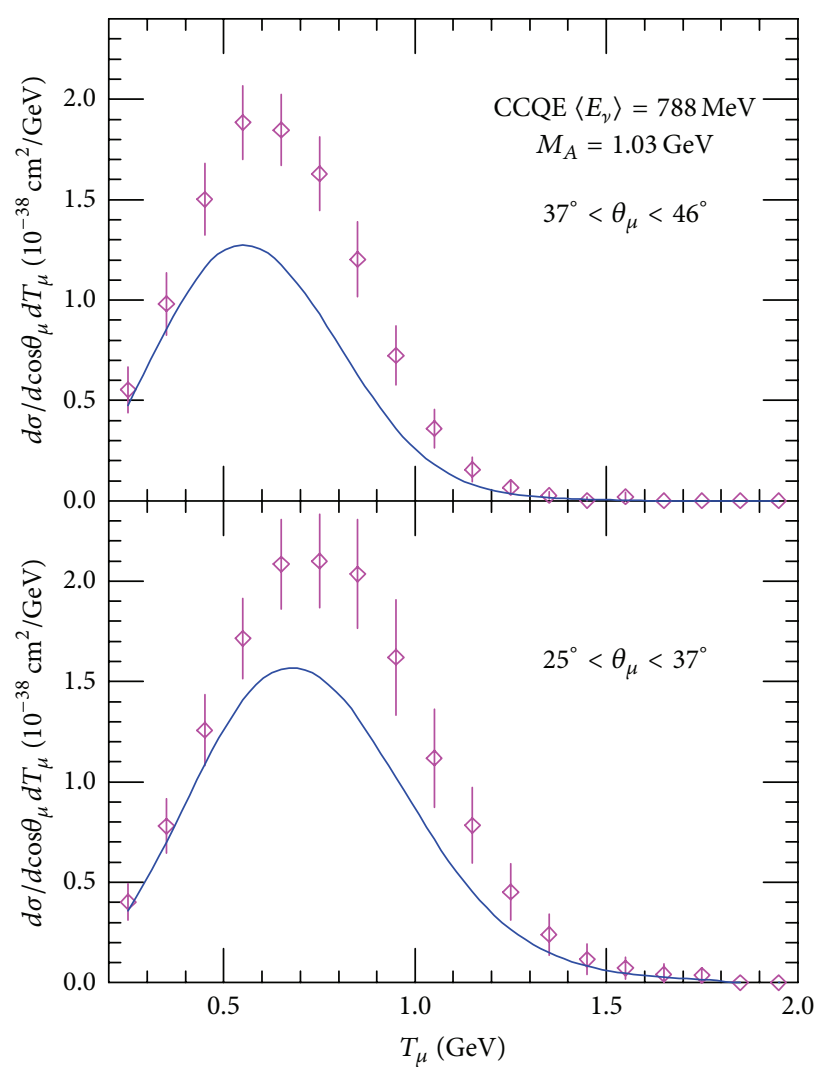

(b)

Figure 4: (a) Inclusive electron-carbon cross-section at beam energy $E_{e}=730 \mathrm{MeV}$ and electron scattering angle $\theta_{e}=37^{\circ}$, plotted as a function of the energy loss $\omega$. The data points are taken from [3]. (b) Flux averaged double differential CCQE cross-section measured by the MiniBooNE collaboration [5], shown as a function of the kinetic energy of the outgoing muon. The upper and lower panels correspond to different values of the muon scattering angle. Theoretical results have been obtained using the same spectral functions and vector form factors employed in the calculation of the electron scattering cross-section of the left panel, and a dipole parameterization of the axial form factor with $M_{A}=1.03 \mathrm{GeV}$.

It has to be pointed out, however, that the approaches of [34-36], while including two-nucleon current contributions, are based on the oversimplified independent particle model (IPM) of nuclear structure, the deficiencies of which have long been recognized (In their classic Nuclear Physics book, first published in 1952, Blatt and Weisskopf warn the reader that "the limitation of any independent particle model lies in its inability to encompass the correlation between the positions and spins of the various particles in the system" [38].) This issue is of paramount importance for the interpretation of CCQE-like events, since within the IPM two particle-two hole states can only be excited by two-nucleon meson-exchange currents (MEC). On the other hand, in the presence of NN correlations final states with two nucleons in the continuum may be also produced by the following additional mechanisms, which do not involve two-nucleon currents: (i) initial state correlations (ISC) and (ii) final state interactions (FSI).

A fully consistent analysis of the role of two particle-two hole final states within a realistic model of nuclear structure obviously requires that all mechanisms leading to the appearance of these final states be included, using a quantummechanical approach that is properly taking into account the interference between the transition amplitudes involving one- and two-nucleon currents. Within the approach of [34-36], based on a model of nuclear dynamics in which correlations are not taken into account, these interference terms are generated by adding "ad hoc" contributions to the two-body current [39].

Within the IA, ISC are taken into account using realistic spectral functions, which include the contribution of the continuum spectrum associated with unbound states of the residual nucleus. Their main effect is the appearance of a tail of the cross-section, extending to large $\omega$, which is clearly visible in Figure 4(a). Semi-inclusive $\left(e, e^{\prime} p\right)$ data [17] suggest that this contribution is not large, amounting to $\sim 10 \%$ of the integrated spectrum. In principle, this reaction mechanism might be clearly identified by detecting two nucleons moving in opposite directions with momenta much larger than that the Fermi momentum $p_{F} \sim 250 \mathrm{MeV}$ (see, e.g., [40]).

In inclusive processes, FSI lead to a shift of the energy loss spectrum, arising from interactions between the knocked out nucleon and the mean field of the recoiling nucleus, and a redistribution of the strength from the quasifree bump to the tails, resulting from rescattering processes. Theoretical studies of electron-nucleus scattering suggest that in the 
kinematical region relevant to the MiniBooNE analysis, the former mechanism, which does not involve the appearance of two particle-two hole final states, dominates. A recent discussion of the inclusion of FSI within the IA scheme can be found in [41]. A different approach, based on a Monte Carlo simulation, is described in [42].

As advocated in [34-36], the most important contribution involving two particle-two hole final states is likely to arise from processes involving MEC, the inclusion of which is long known to be needed to explain the measured nuclear electromagnetic response in the transverse channel [8].

The role of the two nucleon current in electron scattering is best illustrated by comparing the longitudinal and transverse $y$-scaling functions, shown in Figure 5. Scaling in the variable $y$ follows from the dominance of one-nucleon processes, allowing one to write the equation expressing conservation of energy in a very simple form. As a consequence, in the limit of large momentum transfer, the nuclear response, which is in general a function of both $\mathbf{q}$ and $\omega$, becomes a function of a single variable $y=y(\mathbf{q}, \omega)[43$, 44]. The occurrence of scaling provides a strong handle on the reaction mechanism, while the observation of scaling violations reveals the role played by processes beyond the IA.

Figure 5 shows the scaling functions associated with the longitudinal $(\mathrm{L})$ and transverse $(\mathrm{T})$ responses of Carbon, extracted from electron scattering data $[45,46]$. The onset of scaling is clearly visible in the region of the quasifree peak, corresponding to $y \sim 0$, where the data points at different momentum transfer tend to sit on top of one another as $|\mathbf{q}|$ increases. On the other hand, large scaling violations, arising mainly from resonance production, appear in the transverse channel at $y>0$, corresponding to $\omega>Q^{2} / 2 M$. In addition, in the scaling region, the transverse function turns out to be significantly larger than the longitudinal one, while within the IA picture the two scaling functions are expected to be identical.

The results of highly accurate calculations carried out for light nuclei in the nonrelativistic regime strongly suggest that in the quasielastic region, single nucleon knockout processes are dominant in the longitudinal channel, while both one- and two-nucleon mechanisms provide comparable contributions in the transverse channel [47].

The authors of [34-36] carried out extensive calculations of the CCQE neutrino-carbon cross-section, averaged over the MiniBooNE flux, taking into account the effects of MEC as well as collective nuclear excitations, which are known to be important at low momentum transfer. As an example, in Figure 6, the results of these approaches, obtained using a value of the axial mass consistent with the one extracted from deuteron data, are compared to the MiniBooNE muon energy spectrum at muon scattering angle $\theta_{\mu}$ such that $0.8 \leq$ $\cos \theta_{\mu} \leq 0.9$. After the inclusion of MEC, both schemes turn out to provide a quantitative account of the data, and the same pattern is observed for all values of $\theta_{\mu}$.

Figure 7 shows a comparison between MiniBooNE data and the results of a different theoretical approach [48]. The authors of [48] developed a phenomenological procedure based on an extension of the $y$-scaling analysis, suitable to

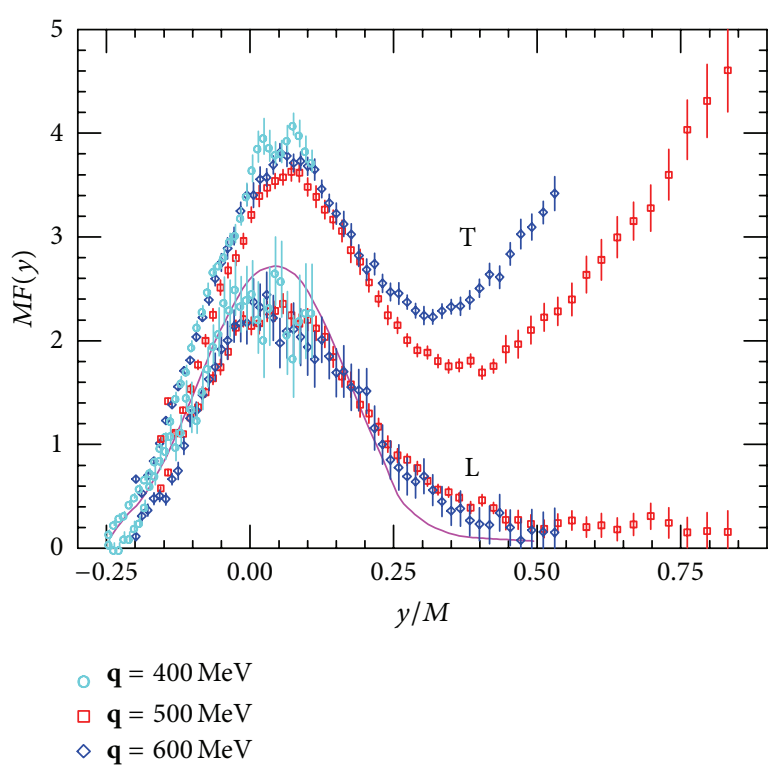

FIGURE 5: Longitudinal (L) and transverse (T) scaling functions of Carbon at $|\mathbf{q}|=400,500$, and $600 \mathrm{MeV}$, resulting from the analysis of [45], based on the data of [46]. For comparison, the solid line shows the scaling function obtained from the IA using the spectral function of [15].

take into account the effects of processes involving MEC. It is apparent that, while at $0.8 \leq \cos \theta_{\mu} \leq 0.9$ (Figure 7(a)), the inclusion of two-nucleon effects brings theory and experiment into agreement, at the larger angles corresponding to $0.3 \leq \cos \theta_{\mu} \leq 0.4$, the measured cross-section is still severely underestimated.

As pointed out above, a fully consistent treatment of processes involving two particle-two hole final states requires a realistic model of nuclear structure, taking into account the effects of NN correlations. Models including MEC within the framework of the IPM, such as those of [34-36], are in fact based on the strong assumption that meson exchange, while playing an important role when the associated current is involved in interactions with an external probe, can be safely ignored in the description of the nuclear initial and final states. The resulting description of the nuclear scattering process appears to be conceptually inconsistent, although the impact of this issue on the numerical results needs to be carefully investigated.

Going beyond this scheme in the kinematical region in which nonrelativistic approximations are not applicable requires an extension of the factorization paradigm underlying the IA, expressed by (7).

The starting point is the generalization of the ansatz of (7) for the hadronic final state to the case in which the interaction with the probe involves two-nucleons as follows:

$$
|X\rangle \longrightarrow\left|\mathbf{p} \mathbf{p}^{\prime}\right\rangle \otimes\left|n_{(A-2)}\right\rangle=\left|n_{(A-2)} ; \mathbf{p p}^{\prime}\right\rangle,
$$

where $\left|n_{(A-2)}\right\rangle$ is the state of the spectator $(A-2)$-nucleon system, carrying momentum $\mathbf{p}_{n}$. 


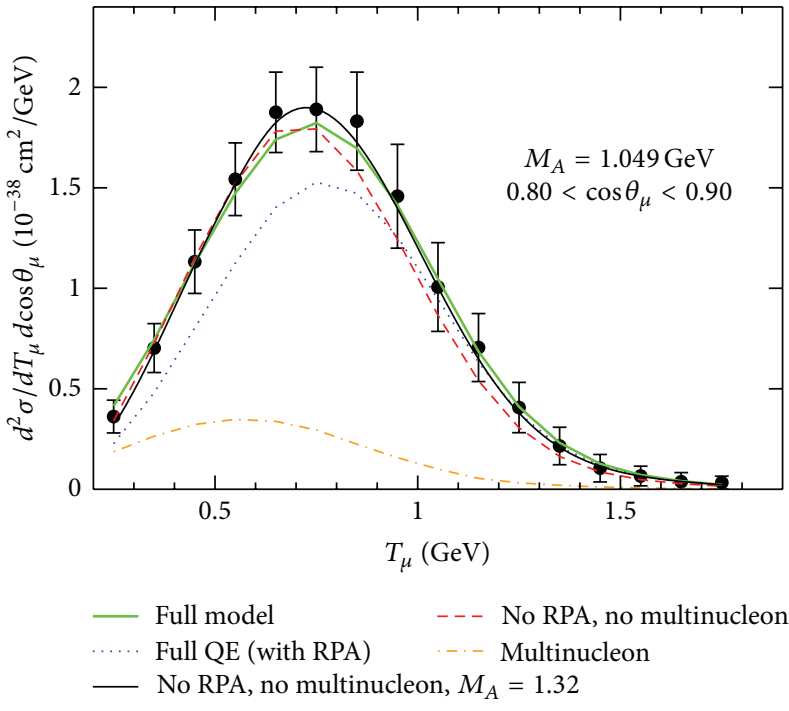

(a)

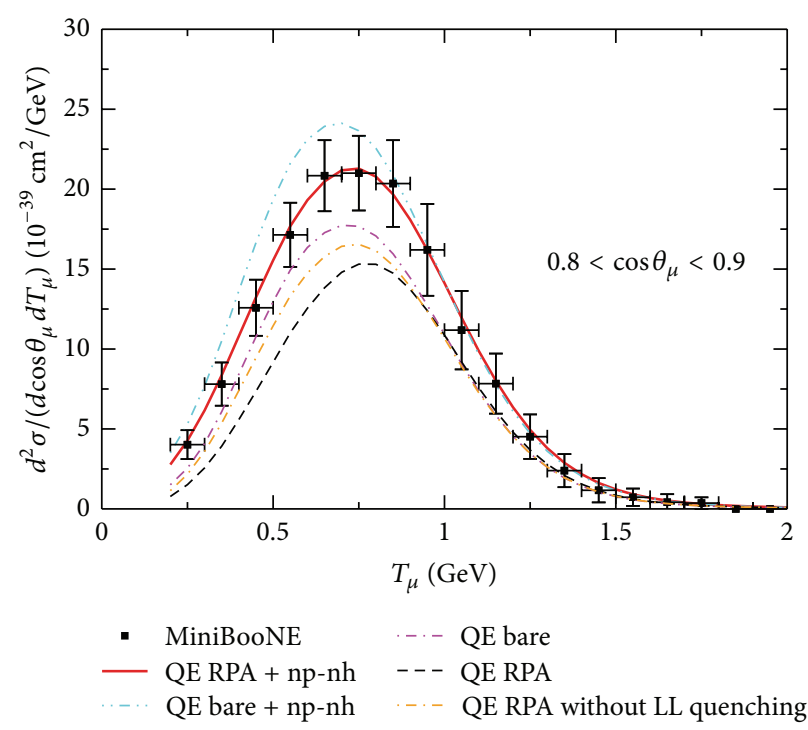

(b)

FIGURE 6: Comparison between the flux averaged muon energy spectrum at muon scattering angle $\theta_{\mu}$ such that $0.8 \leq \cos \theta_{\mu} \leq 0.9$, measured by the MiniBooNE collaboration [5] and the theoretical results of [34, 35] (thick solid line of (b)) and [36] (thick solid line of (a)). All theoretical calculations have been carried out using a value of the axial mass consistent with the one extracted from deuteron data.

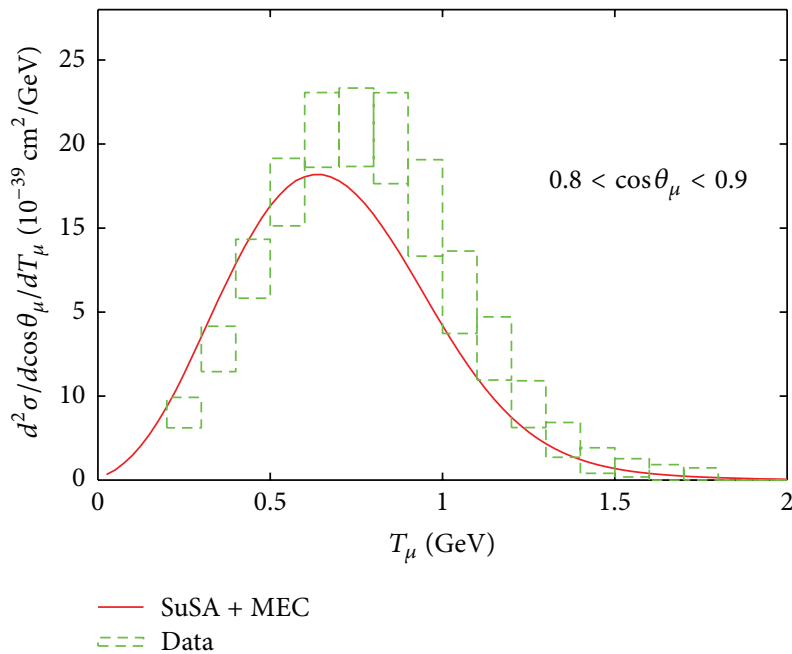

(a)

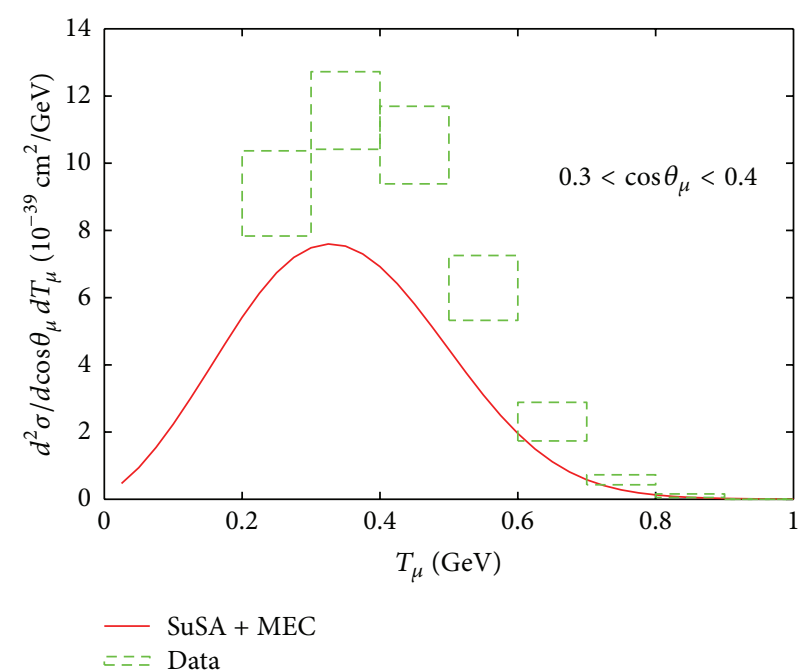

(b)

FIGURE 7: Comparison between the flux averaged muon energy spectra measured by the MiniBooNE collaboration at muon scattering angle $\theta_{\mu}$ such that $0.8 \leq \cos \theta_{\mu} \leq 0.9$ (a) and $0.3 \leq \cos \theta_{\mu} \leq 0.4$ (b) [5] and the results of the approach of [48]. The data, with their error bars, are represented by the boxes, while the solid lines show the calculated spectra.

It follows that the matrix element of the two nucleon current simplifies to (compare to (10))

$$
\begin{aligned}
\left\langle X\left|j_{i j}^{\mu}\right| 0\right\rangle \longrightarrow & \int d^{3} k d^{3} k^{\prime} M_{n}\left(\mathbf{k}, \mathbf{k}^{\prime}\right) \\
& \times\left\langle\mathbf{p} \mathbf{p}^{\prime}\left|j_{i j}^{\mu}\right| \mathbf{k} \mathbf{k}^{\prime}\right\rangle \delta\left(\mathbf{k}+\mathbf{k}^{\prime}-\mathbf{p}_{n}\right),
\end{aligned}
$$

with the amplitude $M_{n}\left(\mathbf{k}, \mathbf{k}^{\prime}\right)$ given by

$$
M_{n}\left(\mathbf{k}, \mathbf{k}^{\prime}\right)=\left\langle n_{(A-2)} ; \mathbf{k k}^{\prime} \mid 0\right\rangle \text {. }
$$

Within this scheme, the nuclear amplitude $M_{n}\left(\mathbf{k}, \mathbf{k}^{\prime}\right)$ turns out to be independent of $\mathbf{q}$, and can therefore be obtained within nonrelativistic many body theory without any problems.

The connection with the spectral function formalism discussed in Section 2.1 becomes apparent noting that the two-nucleon spectral function $P\left(\mathbf{k}, \mathbf{k}^{\prime}, E\right)$, yielding the probability of removing two nucleons from the nuclear ground state leaving the residual system with excitation energy $E$, is defined as

$$
P\left(\mathbf{k}, \mathbf{k}^{\prime}, E\right)=\sum_{n}\left|M_{n}\left(\mathbf{k}, \mathbf{k}^{\prime}\right)\right|^{2} \delta\left(E+E_{0}-E_{n}\right)
$$


where $M_{n}\left(\mathbf{k}, \mathbf{k}^{\prime}\right)$ is defined as in (39) and $E_{0}$ is the ground state energy.

The two-nucleon spectral function of uniform and isospin symmetric nuclear matter at equilibrium density has been calculated within nuclear many-body theory using a realistic hamiltonian [49]. As an example, the resulting relative momentum distribution, defined as

$$
n(\mathbf{Q})=4 \pi|\mathbf{Q}|^{2} \int d^{3} K n\left(\frac{\mathbf{Q}}{2}+\mathbf{K}, \frac{\mathbf{Q}}{2}-\mathbf{K}\right),
$$

with

$$
\begin{aligned}
& n\left(\mathbf{k}, \mathbf{k}^{\prime}\right)=\int d E P\left(\mathbf{k}, \mathbf{k}^{\prime}, E\right), \\
& \mathbf{K}=\mathbf{k}+\mathbf{k}^{\prime}, \quad \mathbf{Q}=\frac{\mathbf{k}-\mathbf{k}^{\prime}}{2}
\end{aligned}
$$

is shown by the solid line of Figure 8. Comparison with the prediction of the Fermi gas model, represented by the dashed line, indicates that correlation effects are sizable. As expected, they lead to a quenching of the peak of the distributions and an enhancement of the high momentum tail.

Using the $(A-1)$ - and $(A-2)$-nucleon amplitudes of $[14,49]$, ISC and MEC contributions to the nuclear crosssections can be calculated in a fully consistent fashion, taking into account interference and using the the fully relativistic expression of two-nucleon current. As pointed out above, the contribution of FSI is not expected to be critical in the kinematical region spanned by MiniBooNE data. However, in principle, they can be also taken into account within the spectral function formalism [41].

\section{Neutrino Energy Reconstruction}

In recent years, nuclear effects in neutrino interactions, while being interesting in their own right, have been mainly studied to appraise their impact on the determination of neutrino oscillations. As an example, in this section, we will discuss the uncertainty on neutrino energy reconstruction arising from the nuclear models employed in data analysis.

Let us consider, for simplicity, two-flavor mixing. The expression of the probability that a neutrino oscillates from flavor $\alpha$ to flavor $\beta$ after travelling a distance $L$

$$
P_{v_{\alpha} \rightarrow v_{\beta}}=\sin ^{2} 2 \theta \sin ^{2}\left(\frac{\Delta m^{2} L}{4 E_{\nu}}\right),
$$

where $\theta$ and $\Delta m^{2}$ are the mixing angle and the squared mass difference, respectively, clearly shows that the accurate determination of the neutrino energy, $E_{v}$, plays a critical role. A wrongly reconstructed $E_{v}$ does in fact result in an incorrect determination of the mixing angle.

The starting point for neutrino energy reconstruction in $v_{\mu} \mathrm{CCQE}$ interactions is the equation expressing the requirement that the scattering process be elastic, that is,

$$
\left(k_{\nu}+p_{n}-k_{\mu}\right)^{2}=M_{p}^{2}
$$

where $k_{\nu} \equiv\left(E_{v}, \mathbf{k}_{\nu}\right)$ and $k_{\mu} \equiv\left(E_{\mu}, \mathbf{k}_{\mu}\right)$ are the four momenta of the incoming neutrino and outgoing muon, respectively,

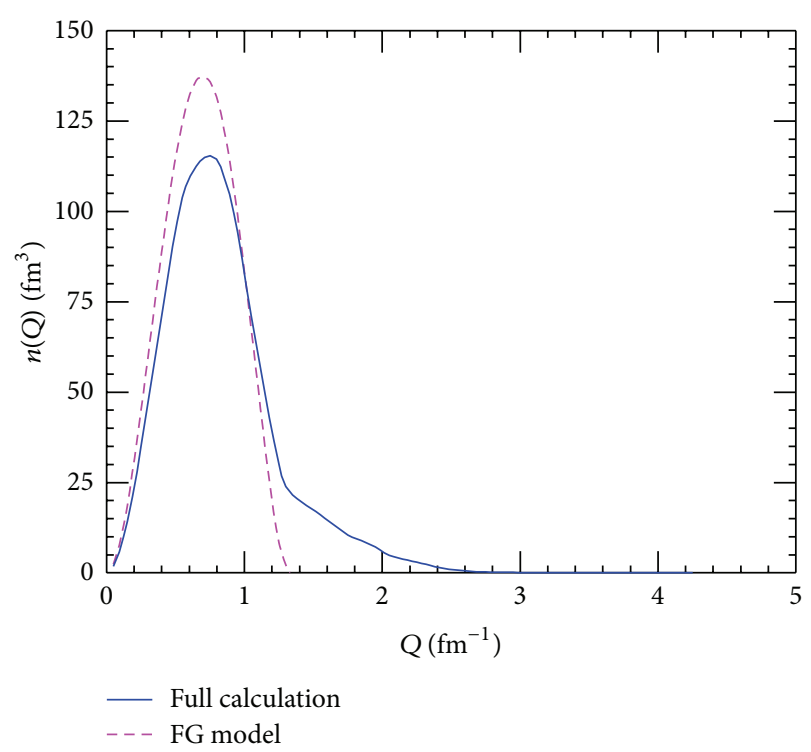

FIGURE 8: Comparison between the two-nucleon relative momentum distribution of nuclear matter computed within nuclear manybody theory using a realistic hamiltonian (solid line) [49], and the prediction of the Fermi gas (FG) model (dashed line).

$M_{p}$ is the proton mass and $p_{n} \equiv\left(E_{n}, \mathbf{p}_{n}\right)$, with $E_{n}=$ $M_{A}-E_{A-1}$ and $E_{A-1}=\sqrt{\left(M_{A}-M_{n}+E\right)^{2}+\left|\mathbf{p}_{n}\right|^{2}}$, is the four momentum struck neutron.

From (44), it follows that

$$
E_{\nu}=\frac{M_{p}^{2}-m_{\mu}^{2}-E_{n}^{2}+2 E_{\mu} E_{n}-2 \mathbf{k}_{\mu} \cdot \mathbf{p}_{n}+\left|\mathbf{p}_{n}\right|^{2}}{2\left(E_{n}-E_{\mu}+\left|\mathbf{k}_{\mu}\right| \cos \theta_{\mu}-\left|\mathbf{p}_{n}\right| \cos \theta_{n}\right)},
$$

where $\theta_{\mu}$ is the muon angle relative to the neutrino beam and $\cos \theta_{n}=\left(\mathbf{k}_{v} \cdot \mathbf{p}_{n}\right) /\left(\left|\mathbf{k}_{v}\right|\left|\mathbf{p}_{n}\right|\right)$. The above equation clearly shows that $E_{v}$ is not uniquely determined by the measured kinematical variables, $E_{\mu}$ and $\theta_{\mu}$, but exhibits a distribution reflecting the energy and momentum distribution of the struck neutron. Therefore, it depends on the nuclear model employed to describe the target ground state.

In the analysis of MiniBooNE data [50], the energy of the incoming neutrino has been reconstructed by setting $\left|\mathbf{p}_{n}\right|=0$ and fixing the neutron removal energy to a constant value, that is, setting $E=\epsilon$, and implying in turn that $E_{n}=M_{n}-\epsilon$. The resulting expression is

$$
E_{v}^{\mathrm{rec}}=\frac{2\left(M_{n}-\epsilon\right) E_{\mu}-\left(\epsilon^{2}-2 M_{n} \epsilon+m_{\mu}^{2}+\Delta M^{2}\right)}{2\left[M_{n}-\epsilon-E_{\mu}+\left|\mathbf{k}_{\mu}\right| \cos \theta_{\mu}\right]},
$$

where $M_{n}$ is the neutron mass, $\Delta M^{2}=M_{n}^{2}-M_{p}^{2}$, and $\left|\mathbf{k}_{\mu}\right|=$ $\sqrt{E_{\mu}^{2}-m_{\mu}^{2}}$ is the magnitude of the three-momentum of the outgoing muon.

In general, the neutrino energy distribution, $F\left(E_{v}\right)$, can be obtained from (45) using values of $\left|\mathbf{p}_{n}\right|$ and $E$ sampled from the probability distribution $\left|\mathbf{p}_{n}\right|^{2} P\left(\mathbf{p}_{n}, E\right)$, and assuming that the polar and azimuthal angles specifying the direction of the neutron momentum be uniformly distributed. 

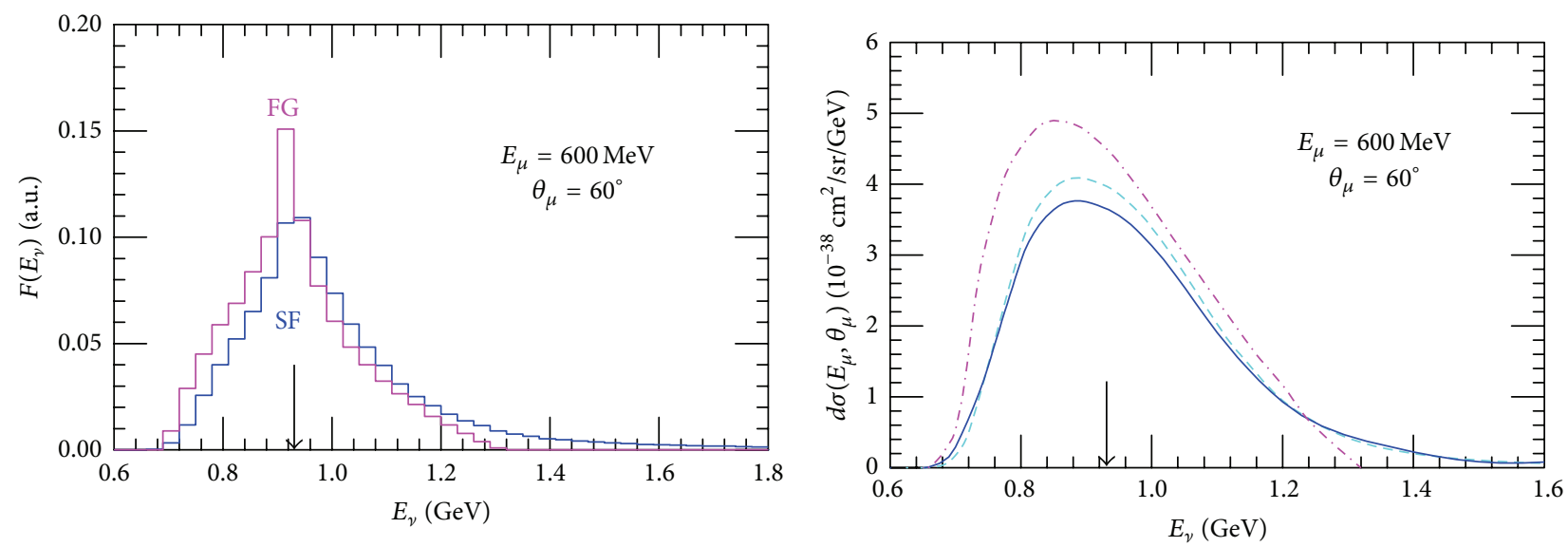

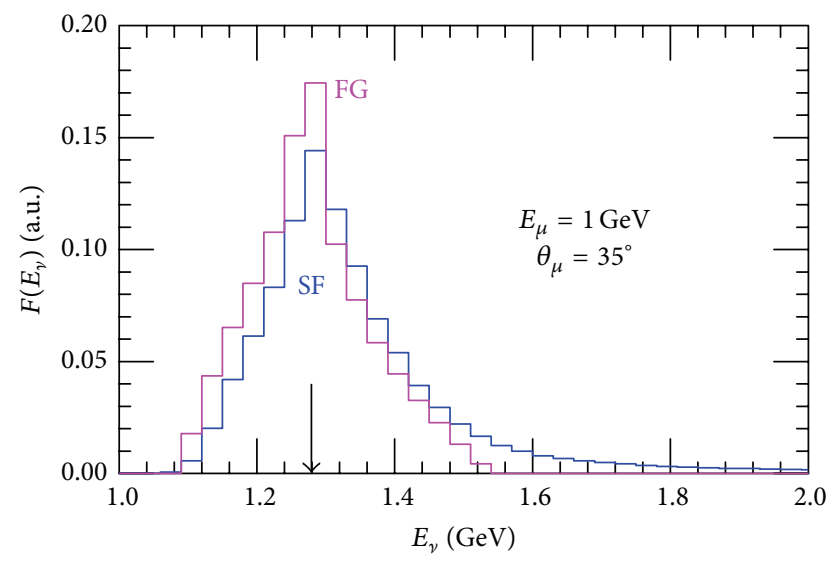

(a)

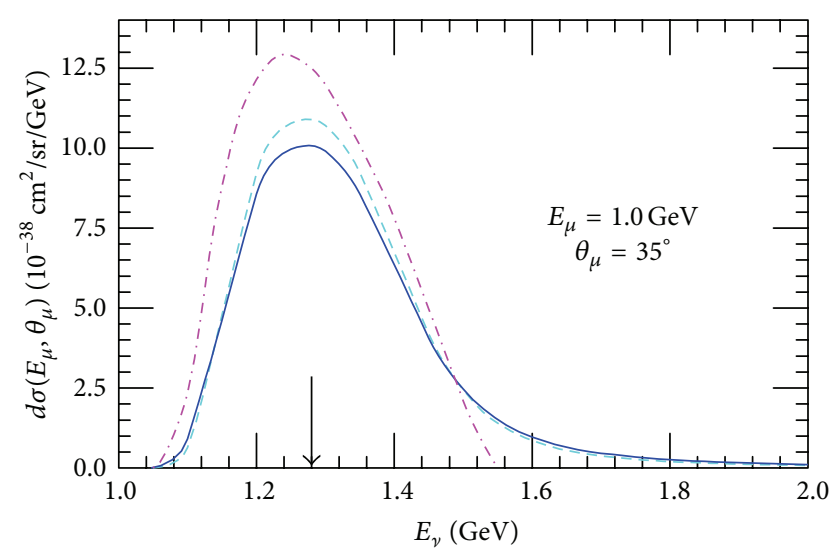

(b)

Figure 9: (a) Neutrino energy distribution at $E_{\mu}=600 \mathrm{MeV}$ and $\theta_{\mu}=60^{\circ}$ (upper panel) and $E_{\mu}=1 \mathrm{GeV}$ and $\theta_{\mu}=35^{\circ}$ (lower panel), reconstructed from (45) using $2 \times 10^{4}$ pairs of $\left(\left|\mathbf{p}_{n}\right|, E\right)$ values sampled from the probability distributions associated with the oxygen spectral function of [49]. (SF) and the RFGM, with Fermi momentum $p_{F}=225 \mathrm{MeV}$ and removal energy $\epsilon=27 \mathrm{MeV}$ (FG). The arrows point to the values of $E_{\nu}^{\text {rec }}$ obtained from (46). (b) Differential cross-section of the process $\nu_{\mu}+A \rightarrow \mu+p+(A-1)$, at $E_{\mu}=600 \mathrm{MeV}$ and $\theta_{\mu}=60^{\circ}$ (upper panel) and $E_{\mu}=1 \mathrm{GeV}$ and $\theta_{\mu}=35^{\circ}$ (lower panel), as a function of the incoming neutrino energy. The solid line shows the results of the full calculation, carried out within the approach of $[15,52]$, whereas the dashed line has been obtained neglecting the effects of FSI. The dot-dash line corresponds to the RFG model with Fermi momentum $p_{F}=225 \mathrm{MeV}$ and removal energy $\epsilon=27 \mathrm{MeV}$. The arrow points to the value of $E_{\nu}^{\text {rec }}$ obtained from (46).

To gauge the effect of the high momentum and high removal energy tails of the spectral functions obtained within realistic dynamical approaches including $\mathrm{NN}$ correlations, the authors of [51] have compared the $F\left(E_{\gamma}\right)$ computed using $2 \times 10^{4}$ pairs of $\left(\left|\mathbf{p}_{n}\right|, E\right)$ values drawn from the probability distributions associated with the oxygen spectral function of [16], to that obtained from the RFGM, with Fermi momentum $p_{F}=225 \mathrm{MeV}$ and removal energy $\epsilon=27 \mathrm{MeV}$. The results corresponding to $E_{\mu}=600 \mathrm{MeV}$ and $\theta_{\mu}=60^{\circ}$, and $E_{\mu}=1 \mathrm{GeV}$ and $\theta_{\mu}=35^{\circ}$, are displayed in Figure 9(a).

The distributions predicted by the RFGM model are more sharply peaked at the neutrino energy given by (46), while the $F\left(E_{\gamma}\right)$ obtained from the spectral function of [15] are shifted towards higher energy by $\sim 20 \mathrm{MeV}$, with respect to the RFG results, and exhibit a tail extending to very large values of $E_{\gamma}$.

Note that the histograms of Figure 9 have been obtained from (45), which in turn follows from the requirement of quasielastic kinematics, (44). However, the reconstruction of the neutrino energy from the measured muon energy and scattering angle is also affected by the occurrence of FSI between the outgoing proton and the spectator nucleons.

In order to assess the total impact of replacing the RFGM with the approach of $[15,52]$, including both ISC and FSI, the authors of [51] have also computed the differential crosssection of the process $\nu_{\mu}+A \rightarrow \mu+p+(A-1)$, as a function of the incoming neutrino energy $E_{v}$, for the muon kinematics of Figure 9(a). The solid lines in Figure 9(b) show the results of the full calculation, carried out using the spectral function of [15], while the dashed lines have been obtained neglecting the effects of FSI and the dot-dash lines correspond to the RFGM. Note that, unlike the histograms of the left panels, the curves displayed in Figure 9(b) have different normalizations.

The differences between the results of the approach obtained from nuclear many-body theory and those of the RFG model turn out to be sizable. The overall shift towards high energies and the tails at large $E_{v}$ appearing 


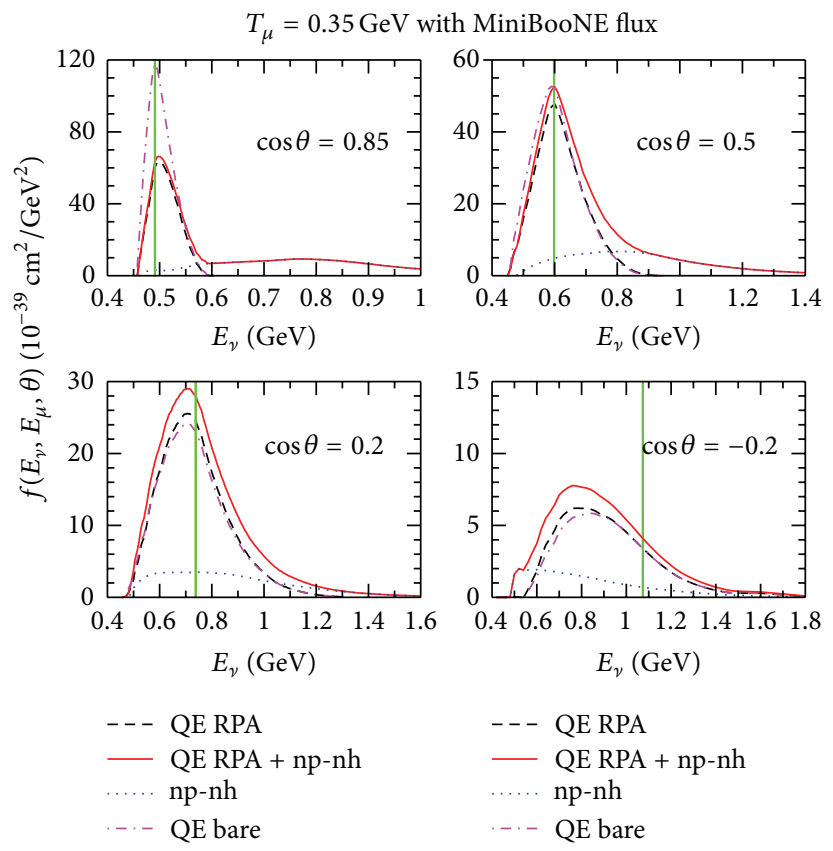

(a)

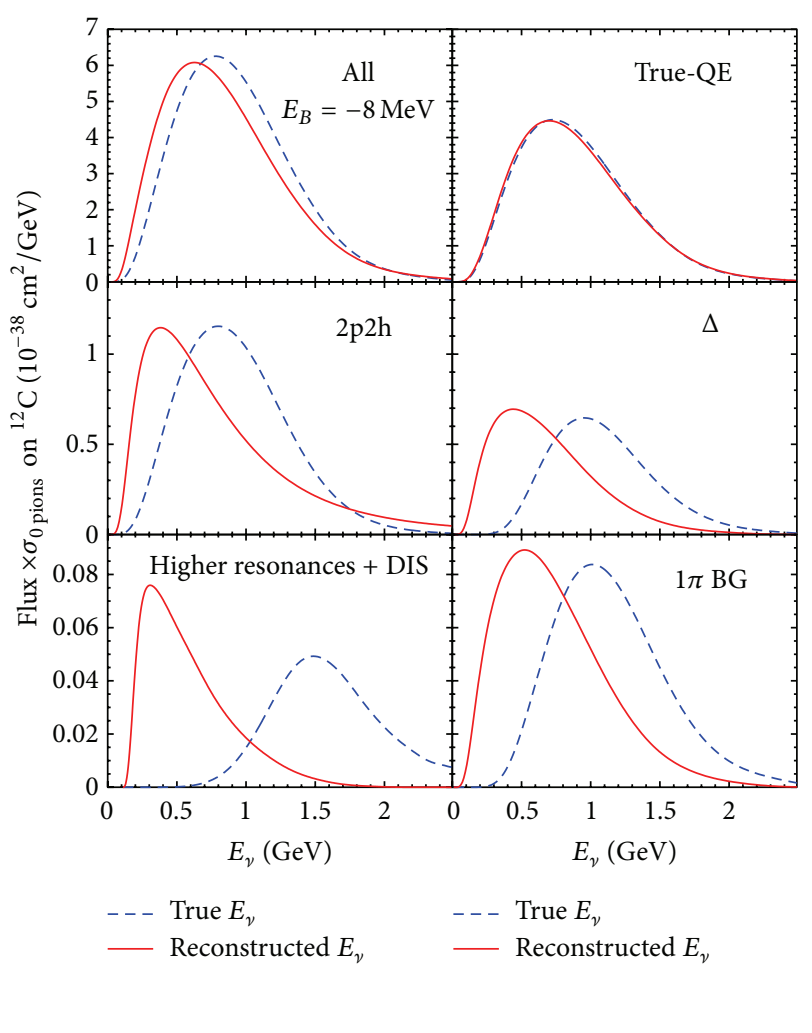

(b)

FIGURE 10: (a) Neutrino energy distributions at $T_{\mu}=350 \mathrm{MeV}$, computed at different muon scattering angles using the MiniBooNE flux [53]. The vertical lines correspond to the reconstructed $E_{\gamma}$ of (46). (b) Event distribution of zero pion events in the MiniBooNE experiment. The dashed curves show the distributions $\Phi\left(E_{\gamma}\right) \sigma_{0 \pi}\left(E_{\gamma}\right)$, defined as in (49), corresponding to different reaction mechanisms, while the distributions $\Phi\left(E_{v}^{\mathrm{rec}}\right) \widetilde{\sigma}_{0 \pi}\left(E_{v}^{\mathrm{rec}}\right)$ are displayed as solid lines [54].

in the histograms of Figure 9(a) are still clearly visible and comparable in size in the cross-section, while the quenching with respect to the RFGM is larger.

The reconstruction of neutrino energy in CCQE-like processes is more complex, as the four-momentum transfer is shared between two nucleons. As a consequence, it requires the knowledge of the two-nucleon spectral function of (39) and (40), and the inclusion of correlation effects is essential.

The uncertainty associated with the reconstruction of $E_{\nu}$ has been recently estimated in [53] taking into account all reaction mechanisms included in the model of $[34,35]$. The authors of [53] computed the neutrino energy distribution, defined by the following equation:

$$
f\left(E_{v}, E_{\mu}, \cos \theta_{\mu}\right) d E_{v}=C\left(\frac{d \sigma}{d E_{\mu} d \cos \theta_{\mu}}\right) \Phi\left(E_{v}\right) d E_{v},
$$

where $\Phi\left(E_{v}\right)$ denotes the flux of incoming neutrinos with energy $E_{v}$ and the normalization constant $C$ is defined through

$$
C^{-1}=\int d E_{\gamma} \Phi\left(E_{\nu}\right) .
$$

The results obtained using the MiniBooNE flux and setting $T_{\mu}=350 \mathrm{MeV}$ are shown in Figure 10(a) for different values of the muon scattering angle. Comparison between the dotdash line, representing the result obtained taking into account single nucleon knock out processes only, and the solid line, corresponding to the full calculation, shows that twonucleon mechanisms are important, and their effect on the neutrino energy reconstruction exhibits a strong dependence on $\cos \theta_{\mu}$.

The impact of reaction mechanisms other than single nucleon knock out on neutrino energy reconstruction has been also analyzed in $[54,55]$. The authors of [54] pointed out that the measured cross-section extracted from events with no pions in the final state-identified as CCQE in both the MiniBooNE and $\mathrm{K} 2 \mathrm{~K}$ analyses-is obtained by dividing the event distribution at a given reconstructed energy by the incoming neutrino flux at the same energy. This quantity, denoted $\widetilde{\sigma}_{0 \pi}\left(E_{v}^{\mathrm{rec}}\right)$, is in general different from the true crosssection evaluated at the true neutrino energy, $\sigma_{0 \pi}\left(E_{\nu}\right)$, which can be obtained from the following relation:

$$
\Phi\left(E_{\nu}\right) \sigma_{0 \pi}\left(E_{\nu}\right)=\int \mathscr{P}\left(E_{\nu} \mid E_{\nu}^{\mathrm{rec}}\right) \Phi\left(E_{\nu}^{\mathrm{rec}}\right) \widetilde{\sigma}_{0 \pi}\left(E_{\nu}^{\mathrm{rec}}\right),
$$

where $\mathscr{P}\left(E_{v} \mid E_{v}^{\text {rec }}\right)$ is the probability density of finding the energy $E_{v}$ in a distribution of events having the same reconstructed energy $E_{\nu}^{\text {rec }}$. 
The right panels of Figure 10 provide an illustration of the main results of [54]. The dashed curves represent the distributions $\Phi\left(E_{\gamma}\right) \sigma_{0 \pi}\left(E_{\gamma}\right)$, defined as in (49), while the distributions $\Phi\left(E_{v}^{\mathrm{rec}}\right) \widetilde{\sigma}_{0 \pi}\left(E_{v}^{\mathrm{rec}}\right)$ are displayed as solid lines. Comparison between different reaction mechanisms shows that for processes involving the two nucleon current the difference between the solid and dashed lines is large. Sizable effects are also visible in the resonance and pion production channels, although these mechanisms turn out to provide smaller contributions to the cross-section.

\section{Summary and Conclusions}

Over the past few years, the availability of the double-diferential CCQE cross-section measured by the MiniBooNE collaboration and the results of a new generation of theoretical studies have led to a better understanding of neutrino-nucleus interactions in a broad kinematical range, as well as to the identification of a number of outstanding unresolved issues.

In view of the fact that no convincing evidence of medium modifications of the nucleon electromagnetic form factors has yet emerged, the excess of CCQE events in carbon reported by the MiniBooNE collaboration [5], the explanation of which within the RFGM requires a large increase of the nucleon axial mass with respect to the value obtained from deuteron data, is likely to be ascribable to the occurrence of processes other than single nucleon knock out, as advocated in [32].

The authors of [34-36] argued that the most important competing mechanism is multinucleon knock out, leading to the appearance of two particle-two hole final states that cannot be distinguished from the one particle-one hole final states associated with single nucleon knockout.

The models developed in [34-36], while proving quite successful in explaining the MiniBooNE neutrino data in terms of processes involving the two-nucleon current, are based on a somewhat oversimplified description of the nuclear initial and final states, in which the effects of correlations are not taken into account. One important consequence of this treatment of nuclear dynamics is that reaction mechanisms triggered by the one-nucleon current and producing two particle-two-hole final states are not taken into account. However, these mechanisms and those involving the twonucleon current are inextricably related to one another and give rise to interference contributions to the cross-section. Therefore, they should be all consistently included.

The main problem associated with a fully consistent description of nuclear structure and dynamics in the kinematical region relevant to neutrino experiments such as MiniBooNE and Minerva stems from the large values of the momentum transfer, which make nonrelativistic approaches inapplicable. The extension of the factorization scheme underlying the spectral function formalism to the case of twonucleon processes may provide a viable approach to overcome this difficulty and study interference effects neglected in the existing calculations.

The dependence of the contribution of processes involving the two-nucleon current on the kinematical conditions should also be carefully investigated. It has been suggested that this analysis may help to shed light on the source of the large disagreement between the values of the nucleon axial mass reported by the MiniBooNE and NOMAD collaborations [56].

The systematic study of the impact of nuclear effects on the determination of neutrino oscillation parameters is still in its infancy $[57,58]$ and is likely to become the most active research field in the coming years. The problem of neutrino energy reconstruction, that plays a critical role in this context, has recently been analyzed using a variety of models including different reaction mechanisms. The emerging picture suggests that the reconstructed energy may turn out to be shifted towards lower values by as much as $\sim 100 \mathrm{MeV}$, with respect to the true energy. The authors of [54] argued that this uncertainty may hamper the extraction of a $\mathrm{CP}$ violating phase from an oscillation result.

\section{Acknowledgments}

The authors declare that some of the results discussed in this paper have been obtained in collaboration with Artur M. Ankowski and Davide Meloni. The authors are also indebted to Camillo Mariani and Makoto Sakuda for many illuminating discussions.

\section{References}

[1] G. P. Zeller, "Highlights from NuInt11," in Proceedings of the 7th International Workshop on Neutrino-Nucleus Interactions in the Few GeV Region (NuInt '11), vol. 1405 of AIP Conference Proceedings, pp. 345-350, Dehradun, India, March 2011.

[2] O. Benhar, D. Day, and I. Sick, "Inclusive quasielastic electronnucleus scattering," Reviews of Modern Physics, vol. 80, no. 1, pp. 189-224, 2008.

[3] J. S. O'Connell, W. R. Dodge, J. W. Lightbody Jr. et al., "Electromagnetic excitation of the delta resonance in nuclei," Physical Review C, vol. 35, no. 3, pp. 1063-1071, 1987.

[4] R. M. Sealock, K. L. Giovanetti, S. T. Thornton et al., "Electroexcitation of the $\Delta(1232)$ in nuclei," Physical Review Letters, vol. 62, no. 12, pp. 1350-1353, 1989.

[5] A. A. Aguilar-Arevalo, C. E. Anderson, A. O. Bazarko et al., "First measurement of the muon neutrino charged current quasielastic double differential cross section," Physical Review $D$, vol. 81, no. 9, Article ID 092005, 22 pages, 2010.

[6] O. Benhar and G. Veneziano, "Nuclear effects in neutral current quasi-elastic neutrino interactions," Physics Letters B, vol. 702, pp. 433-437, 2011.

[7] O. Benhar and D. Meloni, "Total neutrino and antineutrino nuclear cross sections around $1 \mathrm{GeV}$," Nuclear Physics A, vol. 789, no. 1-4, pp. 379-402, 2007.

[8] J. Carlson and R. Schiavilla, "Structure and dynamics of fewnucleon systems," Reviews of Modern Physics, vol. 70, no. 3, pp. 743-841, 1998.

[9] A. Lovato, S. Gandolfi, R. Butler et al., "Charge form factor and sum rules of electromagnetic response functions in ${ }^{12} \mathrm{C}$," Physical Review Letters, vol. 111, no. 9, Article ID 092501, 5 pages, 2013.

[10] G. A. Fiorentini, D. W. Schmitz, P. A. Rodrigues et al., "Measurement of muon neutrino quasi-elastic scattering on a hydrocarbon target at $\mathrm{E}_{v} \sim 3.5 \mathrm{GeV}$," Physical Review Letters, vol. 111, no. 2, Article ID 022502, 6 pages, 2013. 
[11] R. A. Smith and E. J. Moniz, "Neutrino reactions on nuclear targets," Nuclear Physics B, vol. 43, pp. 605-622, 1972.

[12] E. J. Moniz, "Pion electroproduction from nuclei," Physical Review, vol. 184, no. 4, pp. 1154-1161, 1969.

[13] E. J. Moniz, I. Sick, R. R. Whitney, J. R. Ficenec, R. D. Kephart, and W. P. Trower, "Nuclear fermi momenta from quasielastic electron scattering," Physical Review Letters, vol. 26, no. 8, pp. 445-448, 1971.

[14] O. Benhar, A. Fabrocini, and S. Fantoni, "The nucleon spectral function in nuclear matter," Nuclear Physics A, vol. 505, no. 2, pp. 267-299, 1989.

[15] O. Benhar, A. Fabrocini, S. Fantoni, and I. Sick, "Spectral function of finite nuclei and scattering of GeV electrons," Nuclear Physics A, vol. 579, no. 3-4, pp. 493-517, 1994.

[16] O. Benhar, N. Farina, H. Nakamura, M. Sakuda, and R. Seki, "Electron- and neutrino-nucleus scattering in the impulse approximation regime," Physical Review D, vol. 72, no. 5, Article ID 053005, 2005.

[17] D. Rohe, C. S. Armstrong, R. Asaturyan et al., "Correlated strength in the nuclear spectral function," Physical Review Letters, vol. 93, no. 18, Article ID 182501, 2004.

[18] C. F. Perdrisat, V. Punjabi, and M. Vanderhaeghen, "Nucleon electromagnetic form factors," Progress in Particle and Nuclear Physics, vol. 59, no. 2, pp. 694-764, 2007.

[19] V. Bernard, L. Elouadrhiri, and U.-G. Meißner, "Axial structure of the nucleon," Journal of Physics G, vol. 28, no. 1, p. R1, 2002.

[20] H. Budd, A. Bodek, and J. Arrington, "Modeling quasielastic form factors for electron and neutrino scattering," http://arxiv.org/abs/hepex/0308005.

[21] O. Lalakulich and E. A. Paschos, "Resonance production by neutrinos: $J=3 / 2$ resonances," Physical Review $D$, vol. 71, no. 7, Article ID 074003, 2005.

[22] M. Sajjad Athar, S. Chauhan, and S. K. Singh, “Theoretical study of lepton events in the atmospheric neutrino experiments at SuperK," European Physical Journal A, vol. 43, no. 2, pp. 209227, 2010.

[23] T. Leitner, O. Buss, L. Alvarez-Ruso, and U. Mosel, "Electronand neutrino-nucleus scattering from the quasielastic to the resonance region," Physical Review C, vol. 79, no. 3, Article ID 034601, 2009.

[24] P. Berge, H. Burkhardt, F. Dydak et al., "A measurement of differential cross-sections and nucleon structure functions in charged-current neutrino interactions on iron," Zeitschrift für Physik C, vol. 49, no. 2, pp. 187-223, 1991.

[25] R. G. Roberts, The Structure of the Proton, Cambridge University Press, Cambridge, Mass, USA, 1990.

[26] A. Bodek and J. L. Ritchie, "Fermi-motion effects in deepinelastic lepton scattering from nuclear targets," Physical Review D, vol. 23, no. 5, pp. 1070-1091, 1981.

[27] S. A. Kulagin and R. Petti, "Neutrino inclusive inelastic scattering off nuclei," Physical Review D, vol. 76, no. 9, Article ID 094023, 2007.

[28] H. Haider, I. Ruiz Simo, M. Sajjad Athar, and M. J. Vicente Vacas, "Nuclear medium effects in $v(\nu)$-nucleus deep inelastic scattering," Physical Review C, vol. 84, no. 5, Article ID 054610, 2011.

[29] M. Hirai, S. Kumano, and M. Miyama, "Determination of nuclear parton distributions," Physical Review D, vol. 64, no. 3, Article ID 034003, 2001.

[30] R. Gran, E. J. Jeon, E. Aliu et al., "Measurement of the quasielastic axial vector mass in neutrino interactions on oxygen,"
Physical Review D, vol. 74, no. 5, Article ID 052002, 15 pages, 2006.

[31] V. Lyubushkin, B. Popov, J. J. Kim et al., "A study of quasi-elastic muon neutrino and antineutrino scattering in the NOMAD experiment," The European Physical Journal C, vol. 63, pp. 355381, 2009.

[32] O. Benhar, P. Coletti, and D. Meloni, "Electroweak nuclear response in the quasielastic regime," Physical Review Letters, vol. 105, no. 13, Article ID 132301, 2010.

[33] O. Buss, T. Gaitanos, K. Gallmeister et al., "Transporttheoretical description of nuclear reactions," Physics Reports, vol. 512, no. 1-2, pp. 1-124, 2012.

[34] M. Martini, M. Ericson, G. Chanfray, and J. Marteau, "Unified approach fornucleon knock-out and coherent and incoherent pion production in neutrino interactions with nuclei," Physical Review C, vol. 80, no. 6, Article ID 065501, 16 pages, 2009.

[35] M. Martini, M. Ericson, G. Chanfray, and J. Marteau, "Neutrino and antineutrino quasielastic interactions with nuclei," Physical Review C, vol. 81, no. 4, Article ID 045502, 2010.

[36] J. Nieves, I. Ruiz Simo, and M. J. Vicente Vacas, "The nucleon axial mass and the MiniBooNE quasielastic neutrino-nucleus scattering problem," Physics Letters B, vol. 707, no. 1, pp. 72-75, 2012.

[37] R. Gran, J. Nieves, F. Sanchez, and M. J. Vicente Vacas, "Neutrino-nucleus quasi-elastic and $2 \mathrm{p} 2 \mathrm{~h}$ interactions up to 10 GeV," http://arxiv.org/abs/1307.8105 .

[38] J. M. Blatt and V. F. Weisskopf, Theoretical Nuclear Physics, Dover, New York, NY, USA, 1979.

[39] J. E. Amaro, M. B. Barbaro, J. A. Caballero, T. W. Donnelly, and A. Molinari, "Gauge and Lorentz invariant one-pion exchange currents in electron scattering from a relativistic Fermi gas," Physics Report, vol. 368, no. 4, pp. 317-407, 2002.

[40] R. Subedi, R. Shneor, P. Monaghan et al., "Probing cold dense nuclear matter," Science, vol. 320, no. 5882, pp. 1475-1478, 2008.

[41] O. Benhar, "Final-state interactions in the nuclear response at large momentum transfer," Physical Review C, vol. 87, no. 2, Article ID 024606, 8 pages, 2013.

[42] J. Nieves, M. Valverde, and M. J. Vicente Vacas, "Inclusive nucleon emission induced by quasielastic neutrino-nucleus interactions," Physical Review C, vol. 73, no. 2, Article ID 025504, 2006.

[43] G. B. West, "Electron scattering from atoms, nuclei and nucleons," Physics Reports, vol. 18, no. 5, pp. 263-323, 1975.

[44] I. Sick, D. Day, and J. S. McCarthy, "Nuclear high-momentum components and y scaling in electron scattering," Physical Review Letters, vol. 45, no. 11, pp. 871-874, 1980.

[45] J. M. Finn, R. W. Lourie, and B. H. Cottman, "Scaling violation in the separated response functions of C12," Physical Review C, vol. 29, no. 6, pp. 2230-2238, 1984.

[46] P. Barreau, M. Bernheim, J. Duclos et al., "Deep-inelastic electron scattering from carbon," Nuclear Physics A, vol. 402, no. 3, pp. 415-540, 1983.

[47] J. Carlson, J. Jourdan, R. Schiavilla, and I. Sick, "Longitudinal and transverse quasielastic response functions of light nuclei," Physical Review C, vol. 65, no. 2, Article ID 024002, 2002.

[48] J. E. Amaro, M. B. Barbaro, J. A. Caballero, T. W. Donnelly, and C. F. Williamson, "Meson-exchange currents and quasielastic neutrino cross sections in the superscaling approximation model," Physics Letters B, vol. 696, no. 1-2, pp. 151-155, 2011.

[49] O. Benhar and A. Fabrocini, "Two-nucleon spectral function in infinite nuclear matter," Physical Review C, vol. 62, no. 3, Article ID 034304, 2000. 
[50] A. A. Aguilar Arevalo, A. O. Bazarko, S. J. Brice et al., "Measurement of muon neutrino quasielastic scattering on carbon," Physical Review Letters, vol. 100, no. 3, Article ID 032301, 5 pages, 2008.

[51] O. Benhar and D. Meloni, "Impact of nuclear effects on the determination of the nucleon axial mass," Physical Review D, vol. 80, no. 7, Article ID 073003, 6 pages, 2009.

[52] O. Benhar, A. Fabrocini, S. Fantoni, G. A. Miller, V. R. Pandharipande, and I. Sick, "Scattering of GeV electrons by nuclear matter," Physical Review C, vol. 44, no. 6, pp. 2328-2342, 1991.

[53] M. Martini, M. Ericson, and G. Chanfray, "Measurement of the $\overline{t t}$ production cross section in pp collisions at $\sqrt{s}=7 \mathrm{TeV}$ in dilepton final states containing a $\tau$," Physical Review $C$, vol. 85, Article ID 112007, 18 pages, 2012.

[54] O. Lalakulich, U. Mosel, and K. Gellmeister, "Neutrino energy reconstruction in quasielastic-like scattering in the MiniBooNE and T2K experiments," Physical Review C, vol. 86, no. 5, Article ID 054606, 12 pages, 2012.

[55] J. Nieves, F. Sánchez, I. Ruiz Simo, and M. J. Vicente Vacas, "Neutrino energy reconstruction and the shape of the charged current quasielastic-like total cross section," Physical Review D, vol. 85, no. 11, Article ID 113008, 9 pages, 2012.

[56] O. Benhar, "Confronting electron- and neutrino-nucleus scattering," Journal of Physics: Conference Series, vol. 408, Article ID 012042, 2013.

[57] D. Meloni and M. Martini, "Revisiting the T2K data using different models for the neutrino-nucleus cross sections," Physics Letters B, vol. 716, no. 1, pp. 186-192, 2012.

[58] P. Coloma and P. Huber, "Impact of nuclear effects on the extraction of oscillation parameters," http://arxiv.org/abs/1307.1243 . 

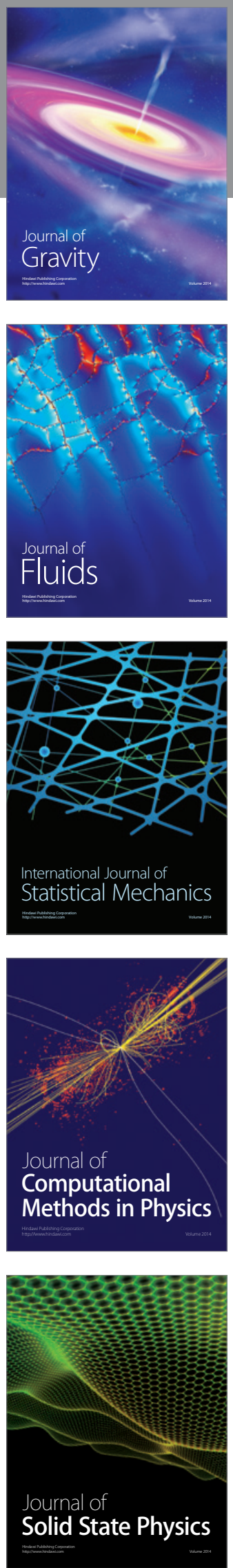

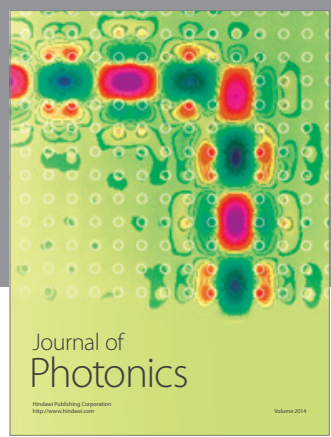

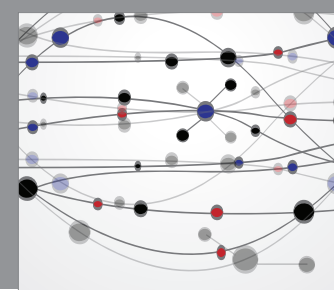

The Scientific World Journal

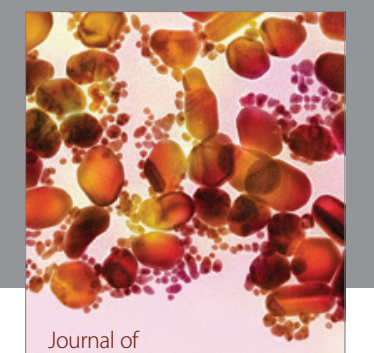

Soft Matter
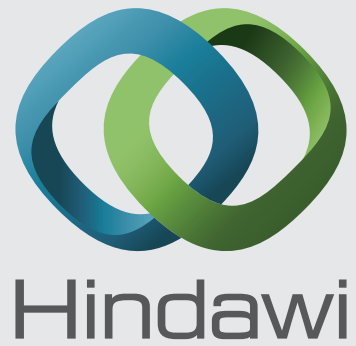

Submit your manuscripts at

http://www.hindawi.com
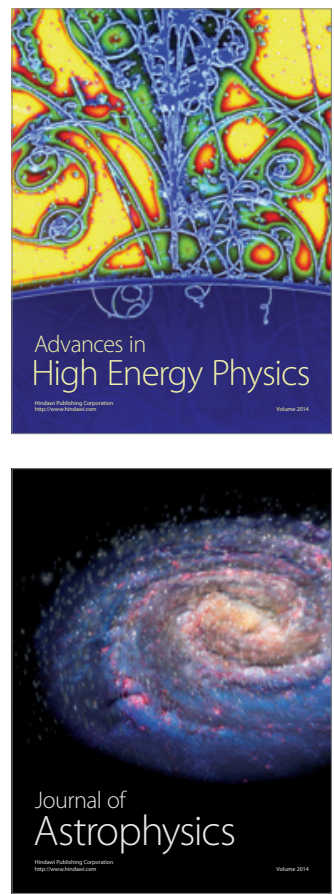
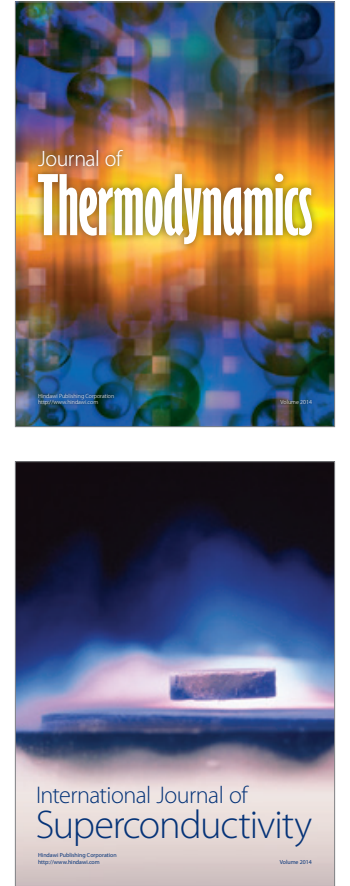
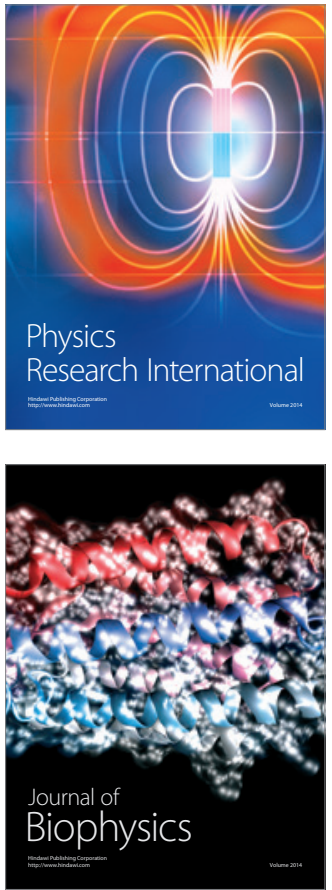
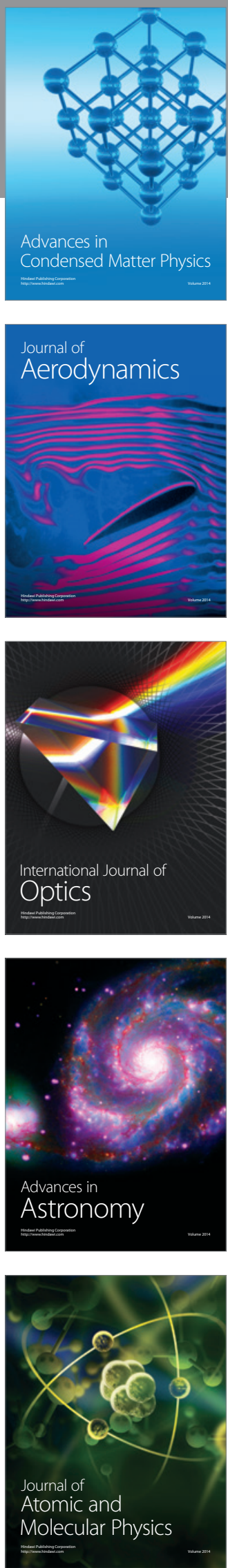